\title{
Nonlinear and long-term beam dynamics in low energy storage rings
}

\author{
A. I. Papash, ${ }^{1,2, *, \dagger}$ A. V. Smirnov, ${ }^{1, \dagger}$ and C. P. Welsch ${ }^{3}$ \\ ${ }^{1}$ Max Planck Institute for Nuclear Physics, Heidelberg, Germany \\ ${ }^{2}$ Karlsruhe Institute of Technology, Karlsruhe, Germany \\ ${ }^{3}$ Cockcroft Institute and The University of Liverpool, United Kingdom
}

(Received 7 February 2013; published 3 June 2013)

\begin{abstract}
Electrostatic storage rings operate at very low energies in the $\mathrm{keV}$ range and have proven to be invaluable tools for atomic and molecular physics. Because of the mass independence of electric rigidity, these machines are able to store a wide range of different particles, from light ions to heavy singly charged biomolecules, opening up unique research opportunities. However, earlier measurements have shown strong limitations in maximum beam intensity, fast decay of the stored ion current, and reduced beam lifetime. The nature of these effects has not been fully understood and an improved understanding of the physical processes influencing beam motion and stability in such rings is needed. In this paper, a comprehensive study into nonlinear and long-term beam dynamics studies is presented on the examples of a number of existing and planned electrostatic storage rings using the BETACOOL, OPERA-3D, and MAD-X simulation software. A detailed investigation into ion kinetics, under consideration of effects from electron cooling and multiple scattering of the beam on a supersonic gas jet target, is carried out and yields a consistent explanation of the physical effects in a whole class of storage rings. The lifetime, equilibrium momentum spread, and equilibrium lateral spread during collisions with the target are estimated. In addition, the results from experiments at the Test Storage Ring, where a low-intensity beam of $\mathrm{CF}^{+}$ions at $93 \mathrm{keV} / \mathrm{u}$ has been shrunk to extremely small dimensions, are reproduced. Based on these simulations, the conditions for stable ring operation with an extremely low-emittance beam are presented. Finally, results from studies into the interaction of 3-30 keV ions with a gas jet target are summarized.
\end{abstract}

DOI: 10.1103/PhysRevSTAB.16.060101

PACS numbers: 29.20.db, 37.10.Mn

\section{INTRODUCTION}

Electrostatic storage rings are able to store beams of charge particles at very low energies between some $\mathrm{eV} \cdot q$ and a few hundreds of $\mathrm{eV} \cdot q$, where $q$ denotes their charge state. These storage devices have been developed alongside ion traps in response to the needs of the astrophysics, atomic physics, and molecular physics communities [1,2]. They rely on electrostatic bending and focusing elements rather than the bending magnets and focusing magnetic quadrupoles used in magnetic storage rings. The main difference between magnetic and electrostatic storage rings is that the fields in the latter are mass independent and hence there is no upper limit on the particle mass that can be stored. For magnetic storage rings, the magnetic rigidity depends on the ion charge to ion mass ratio $q / A$. In electrostatic rings, the electric rigidity and voltage applied to the electrostatic bending elements are

\footnotetext{
*alexander.papash@kit.edu

On leave from Joint Institute for Nuclear Research, Dubna, Russia.

Published by the American Physical Society under the terms of the Creative Commons Attribution 3.0 License. Further distribution of this work must maintain attribution to the author(s) and the published article's title, journal citation, and DOI.
}

proportional to the ion kinetic energy $T$ and ion charge $q$ but do not depend on the atomic number $A$. Compared to magnetic storage rings, they have essentially lower limits for beam energy because of the absence of eddy currents and hysteresis in bending elements. In addition, a so-called reaction microscope for the study of high-precision effects in collisions between an ultrasonic gas jet target and the stored particle beam, integrated in such low-energy storage ring, promises access to hitherto impossible experimental studies [3].

Despite their distinct advantages for fundamental research in the low-energy regime, few electrostatic ion storage rings are currently in operation around the world. Those that are in use all have a comparable compact racetrack-shaped layout and work at a fixed energy of 20 $[4,5]$ or $30 \mathrm{keV} \cdot q$ [6] with a continuous beam. A double electrostatic ring, operating in a merged beam configuration and at temperatures below $20 \mathrm{~K}$, is presently being commissioned at the Manne Siegbahn Laboratory in Stockholm, Sweden [7]. In addition, a fixed energy storage ring for energies up to $50 \mathrm{keV} \cdot q$ has been designed, assembled and tested at the University of Frankfurt, Germany [8,9], a cryogenic storage ring (CSR) is being commissioned at the Max Planck Institute for Nuclear Physics in Heidelberg, Germany [10], and a fixed energy ring for beam energies of up to $30 \mathrm{keV} \cdot q$ has been designed by researchers at 
KACST in Saudi Arabia in collaboration with the authors of this paper $[11,12]$.

Beam parameters measured in operating electrostatic rings include emittance, beam profile, beam current loss rate, and energy spread, see e.g. [4-6,13,14]. Strong limitations on beam intensity and reduced lifetime have been indicated and ion losses have been shown to be highly dependent on the lattice structure of the storage ring and beam current [15]. The nature of these effects has not been fully understood yet.

Possible causes of these limits to beam current and lifetime proposed to date $[16,17]$ include nonlinear fields in the electrostatic elements, parametric resonances due to modulation of the space charge tune shift, as well as coupling between the longitudinal and transverse particle motion. It has already been noted that excessive beam losses occur at beam intensities too low to be explained by a negative shift of the betatron tune due to space charge or parametric resonances.

An improved understanding of beam behaviorespecially nonlinear effects, long-term beam dynamics, ion kinetics, and ion equilibrium conditions-in lowenergy storage rings will pave the way for successful operation and optimization of these machines. Therefore, additional studies have been initiated to improve knowledge of the nature of beam losses and the physics of processes in ultra-low-energy storage rings.

\section{ACCEPTANCE AND BETATRON TUNE SHIFT IN ELISA RING}

We have carried out simulations of the beam behavior including nonlinear terms in the equations of motion derived from a harmonic Fourier analysis of the electric field distribution in the electrostatic deflectors. We have also tracked ions in a full 3D computer model of the ring using relaxation maps of the electric field distribution in the bending and focusing elements. Finally, we have investigated transition processes, equilibrium conditions, and long-term beam dynamics based on the kinetic equations. On this basis we propose a consistent explanation of effects previously observed in electrostatic storage rings.
In order to verify our computer studies we chose ELISA, the first electrostatic ring dedicated to atomic physics experiments and in operation at Aarhus University since the late 1990s [4], as an example. The layout of the ELISA ring is illustrated in Fig. 1. In this ring two $160^{\circ}$ electrostatic deflectors and two $10^{\circ}$ parallel plate deflectors, together with four electrostatic quadrupole doublets, form a simple racetrack structure. Split deflectors allow neutral particles to be detected at the end of the straight sections. Rings with a similar lattice geometry have been built at KEK in Japan [5] and at Tokyo Metropolitan University, Japan [6]. It should be noted that spherical-shaped deflectors were used in the original design of ELISA to provide equal focusing in both horizontal and vertical planes [13] but have later been replaced by electrostatic deflectors of cylindrical shape.

Systematic experimental studies of beam parameters in ELISA showed strong limitations on the beam current and a reduced beam lifetime. As an example, the measured decay of a stored beam of negative oxygen ions at $22 \mathrm{keV}$ is presented in Fig. 2(a). It can be seen that the ion losses are highly dependent on beam intensity, especially for the ring using spherical deflectors.

The theory of ion motion in electrostatic bending and focusing elements is well developed, see e.g. [17-20]. The expressions for particle oscillations in a spherical deflector might be written as

$$
\begin{array}{r}
x^{\prime \prime}+\frac{1}{R_{\mathrm{eq}}^{2}} x-\frac{1}{R_{\mathrm{eq}}^{3}} x^{2}-\frac{3}{2 R_{\mathrm{eq}}^{3}} y^{2}=0, \\
y^{\prime \prime}+\frac{1}{R_{\mathrm{eq}}^{2}} y-\frac{3}{R_{\mathrm{eq}}^{3}} x y=0 .
\end{array}
$$

Second order equations of motion for a spherical deflector include sextupole components as well as coupling between radial and vertical planes. In linear approximation a spherical deflector provides equal focusing in both horizontal and vertical planes.

The motion in a cylindrical deflector is described by the second order equations

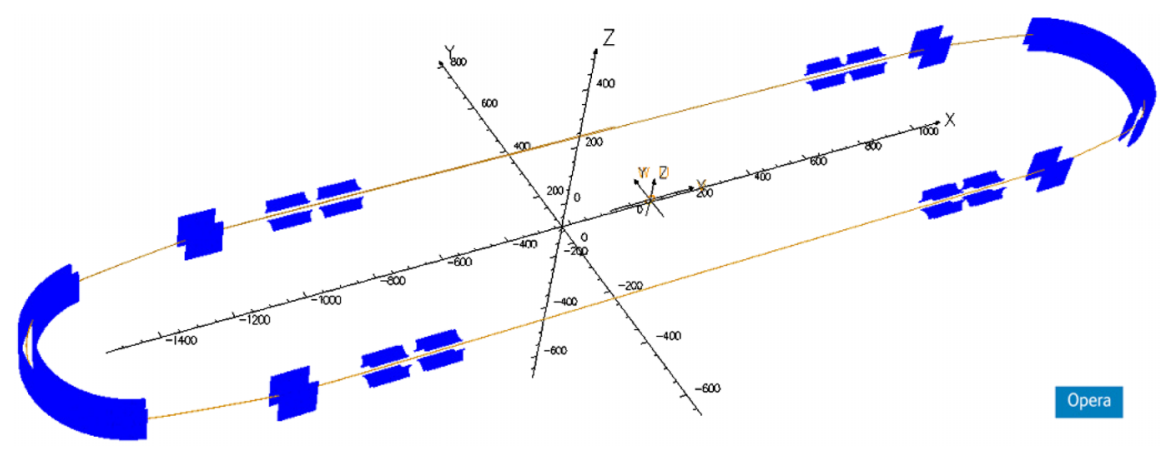

FIG. 1. Model of the ELISA ring in OPERA-3D. The dimensions and distances were taken from [4,13]. Particles were tracked over many turns in order to find a stable solution for the closed equilibrium orbit. 


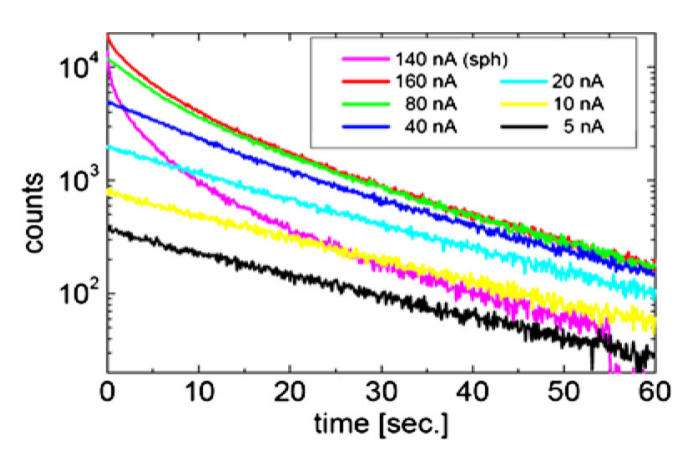

(a)

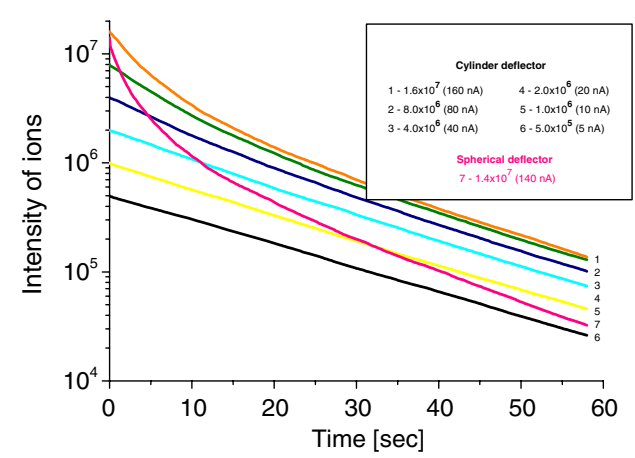

(b)

FIG. 2. Decay of stored $\mathrm{O}^{-}$ions in ELISA at $22 \mathrm{keV}$ beam energy: (a) experimental data, from [15]; (b) BETACOOL simulations [26,27]. The pink curve shows the drop of the beam current in the ELISA ring with electrodes of spherical shape; all remaining data refer to the ELISA ring equipped with cylindrical deflectors.

$$
x^{\prime \prime}+\frac{2}{R_{\mathrm{eq}}^{2}} x-\frac{1}{R_{\mathrm{eq}}^{3}} x^{2}=0, \quad y^{\prime \prime}=0 .
$$

The sextupole component for a cylindrical deflector is smaller than that for a spherical deflector and affects only the horizontal plane. There is also no coupling between horizontal and vertical motion in a cylindrical deflector, at least up to the second order. The focusing strength of a cylindrical deflector in the horizontal (bending) direction is twice that of a spherical one. In the vertical direction, a cylindrical deflector might be considered as a drift.

In order to study ion motion and nonlinear effects we created a model of the ELISA ring in the commercial OPERA-3D field simulation software [21], see Fig. 1. This code package incorporates a number of different modules including the electrostatic 3D field solver TOSCA3D and SCALA3D programs and allows for beam tracking under the influence of space charge effects. In order to benchmark earlier experimental data, two different maps were created with both spherical and cylindrical deflectors implemented for the main bending elements. The ELISA ring model was then searched for a closed orbit by tracking ions through the full 3D electric fields over many revolutions.

The linear ion optics parameters of ELISA, such as its beta functions, betatron tunes, and dispersion function, were obtained from the TRACE-3D [22] and MAD-X [23] simulation codes. We incorporated linear transfer matrices for all electrostatic elements into MAD-X because the program does not include such deflectors as predefined ion optical element. At low energies $(\beta \ll 1)$ the linear transfer matrix $\mathfrak{R}$ of electrostatic deflector with bending angle $\theta$, bending radius $R$, and effective length $L=R \cdot \theta$ might be written in the nonrelativistic form [19,20]

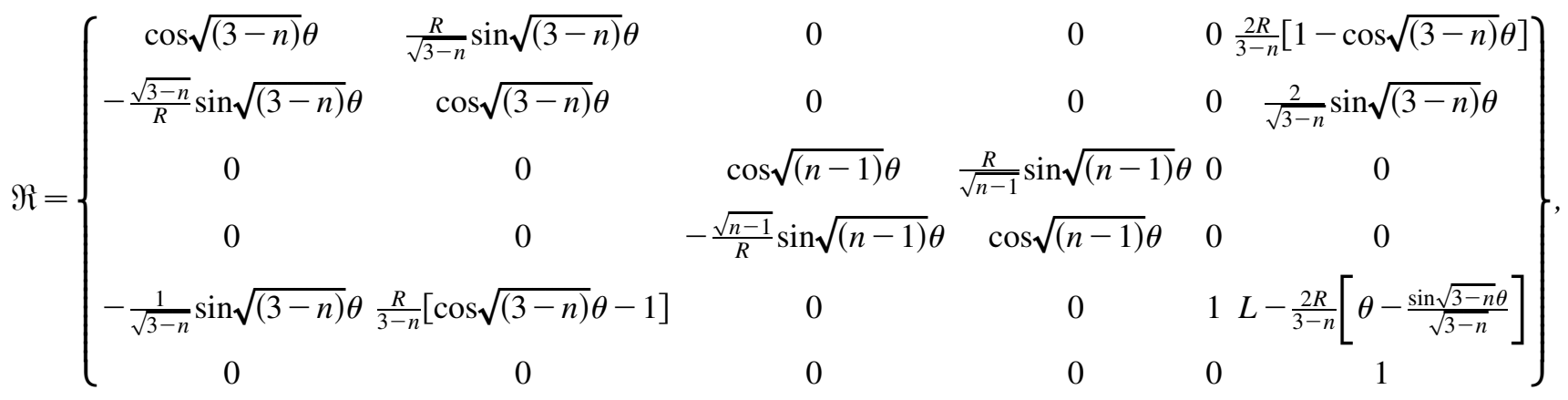

where the electric field index $n=1$ for a cylindrical deflector and $n=2$ for a spherical deflector.

The periodicity of ELISA is two and its original lattice with spherical deflectors showed a strong waist in the horizontal plane in the middle of the $160^{\circ}$ spherical deflector, where $\beta_{x}=0.14 \mathrm{~m}$ [4]. Also, there were two instances of a sharp minimum of the vertical beta function $\beta_{y}=0.1 \mathrm{~m}$ inside the $160^{\circ}$ deflector due to the strong focusing of spherical shape electrodes in the vertical plane [24,25]. When the spherical deflector was replaced by cylindrical electrodes, acting as a drift in the vertical direction, it was possible to vary the vertical beta function inside the $160^{\circ}$ deflector between 0.2 and $2 \mathrm{~m}$. However, the higher value of the beta function in the middle of the 
TABLE I. Integral electric field harmonics of cylindrical and spherical deflectors. Data are normalized to the main dipole component $n=1$.

\begin{tabular}{lcccccc}
\hline \hline & 1 & 2 & 3 & 4 & 5 & 6 \\
$n$ & Dipole & Quadrupole & Sextupole & Octupole & Decapole & Hexapole \\
\hline ESD-CYL & 1 & $5.6 \times 10^{-2}$ & $1.7 \times 10^{-3}$ & $4.3 \times 10^{-5}$ & $2.7 \times 10^{-5}$ & $1.9 \times 10^{-5}$ \\
ESD-SPH & 1 & $1.1 \times 10^{-1}$ & $6.1 \times 10^{-3}$ & $1.3 \times 10^{-3}$ & $3.4 \times 10^{-4}$ & $1.0 \times 10^{-4}$ \\
\hline \hline
\end{tabular}

cylindrical electrode was accompanied by a reduction of the vertical betatron tune to $\nu_{z}<1$. With early ELISA tests having indicated that regimes with a vertical tune of less than one were unstable, ring operation with $160^{\circ}$ cylindrical deflectors was performed with a vertical waist $\beta_{y}=$ $0.4 \mathrm{~m}$ in the middle of the cylindrical deflector and two strong focal minimums in the horizontal plane $\beta_{x}=0.1 \mathrm{~m}$ inside the deflector $[15,24,25]$.

For further analysis we implemented sextupole, octupole, and higher order components of the electric field distribution as aberration factors for a stable analytical solution. The field integrals were calculated along trajectories inside the deflectors at a distance of $14 \mathrm{~mm}$ from the equilibrium orbit where the gap between the electrodes inside the $160^{\circ}$ deflector is $30 \mathrm{~mm}$. Fourier analyses of the field integrals were then performed using OPERA-3D/ TOSCA3D. The resulting amplitudes of the electric field harmonics, normalized to the main dipole component $(n=1)$, are listed in Table I. It is noted that the sextupole and octupole components of the electric field distribution in the spherical deflector is a few times larger than in the cylindrical deflector. Such strong sextupole and octupole components lead to a reduced region of stable betatron motion and large spread of betatron tune in the spherical deflector [24].

We then tracked particles through the ring to determine the dynamic aperture of ELISA. The higher order Fourier components of the electric field distribution were treated as field errors and implemented over the total length of each deflector as a set of thin multipole elements, spaced at equal azimuth steps. We found that all nonlinear terms reduced the ring acceptance and that the sextupole component was the most important. As a result the dynamic aperture of the ring with spherical-shaped deflectors was found to be significantly smaller than of the ring with cylindrical deflectors, especially in the vertical direction [24].

In addition, the amplitude dependence of the betatron tunes in the model of the ELISA ring has been studied. We used fast Fourier transformation analysis of the phase space profile of the tracked beam and derived the fractional part of the betatron tune [25]. Figure 3(a) shows the fractional part of the betatron tunes and one can see that the tune spread for the ring with spherical deflectors is much larger than that for the ring with cylindrical deflectors. For

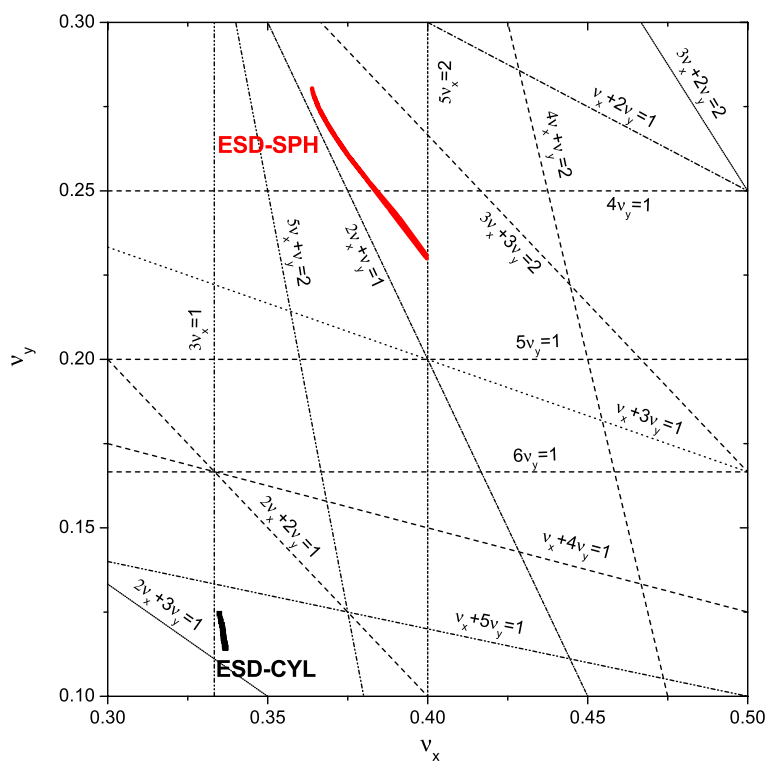

(a)

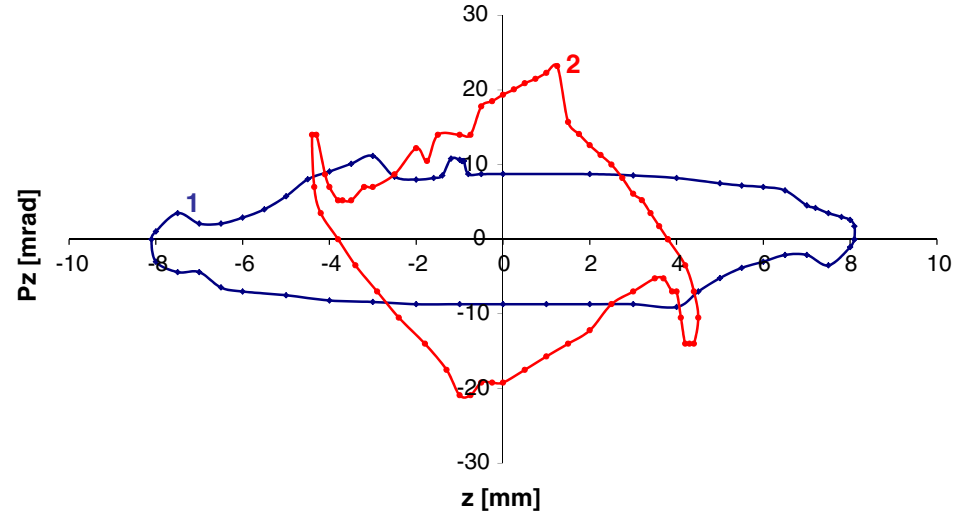

(b)

FIG. 3. Parameters of the ELISA ring: (a) amplitude dependent tune shift in the ring with deflectors of spherical (red) and cylindrical (black) shape, simulated by MAD-X [23]; (b) vertical acceptance of the ELISA ring. Stable orbits have been tracked in relaxation electric field mesh generated by OPERA-3D /TOSCA3D. The middle of the long straight section is marked as (1) and the middle of the $160^{\circ}$ cylinder deflector as (2). The ring acceptance in vertical phase space is less than $30 \pi \mathrm{mm} \mathrm{mrad}$ [22]. 
a span of radial oscillations at $\pm 10 \mathrm{~mm}$, the horizontal betatron tune spread is $3 \times 10^{-3}$ in the case of the ring with cylindrical deflectors, and for the ring with spherical deflectors the tune spread is $3.5 \times 10^{-2}$. The betatron tune spread in the vertical direction is $3.5 \times 10^{-3}$ for the cylindrical deflector and $4 \times 10^{-2}$ for the spherical one. The betatron tune of the ring with spherical deflectors is close to a third order resonance and crosses higher order resonances, in agreement with estimations provided in [16].

Finally, the ring acceptance in the radial and axial phase spaces was estimated by tracking the beam over many revolutions through the relaxation electric field grid generated by OPERA-3D. The initial position and momentum of ions with respect to the reference orbit were varied over a wide range to find the maximum amplitude of stable betatron oscillations. It was found that the acceptance in the radial plane was less than the ring acceptance determined by the available mechanical aperture in the focusing elements. Also, the ring acceptance in the vertical plane was less than $A_{x}<30 \pi \mathrm{mm}$ mrad, see Fig. 3(b), i.e., a few times smaller than would be expected from geometric considerations [24]. For spherical deflectors, the acceptances in both planes were found to be even more limited and thus the effects more pronounced [25]. In the following, we present results from a study into the main forces that drive low-energy ions to the periphery of the reduced ring acceptance and hence lead to beam loss.

\section{ION KINETICS AND LONG-TERM BEAM DYNAMICS}

In order to find the driving forces responsible for pushing ions out of the aperture of the ELISA ring, as well as for a reduced beam lifetime, ion intensity losses, and beam degradation, we have studied transition processes and equilibrium conditions in low-energy storage rings. We have derived growth rates of root mean square (rms) beam emittance and rms momentum spread by simulating the evolution of the ion distribution function with time, using the BETACOOL code to study the long-term beam dynamics in storage rings [26,27]. Initially developed by JINR scientists in collaboration with leading scientific centers worldwide to estimate heating and cooling effects in high-energy storage rings with electron cooling, BETACOOL has been successfully benchmarked at different accelerator labs worldwide [28-37]. The software is under continual development and new physics models in the lowenergy range were implemented for our particular studies. Existing applications include the study of electron, stochastic, gated stochastic and laser cooling methods, as well as the interaction of circulating ions with residual gas, interaction with different kinds of internal targets, intrabeam scattering, beam-beam collisions, external heating of the ion beam, different kinds of particle losses, and barrier rf bucket regimes.
In general, BETACOOL is intended to simulate long-term beam dynamics (in comparison with the ion revolution period in a ring), leading to a variation of the ion distribution function in six-dimensional phase space. The betatron motion inside a storage ring is supposed to be stable and it is treated in linear approximation. Nonlinear effects strongly restrict the amplitude of stable betatron oscillations in storage rings. In our studies we took these nonlinear effects into account by variation of the ring acceptance as input parameter in BETACOOL $[38,39]$.

\section{A. Kinetic equations}

Studies of ion kinetics in storage rings are based on the application of stochastic (kinetic) differential equations to periodic motion. It is assumed that diffusion (i.e. heating processes) and friction (i.e. cooling processes) leading to the growth, reduction or equilibrium of beam phase spaces are small with respect to electromagnetic forces from external bending and focusing elements. The evolution of the ion distribution function is described by the FokkerPlanck equation with friction and diffusion forces of stochastic nature [40]. The main methods of solving stochastic differential equations are based on the transformation of the initial kinetic equation to the form of the Fokker-Planck equation and finding a solution for the density distribution probability function. In some cases, when the effects acting on the distribution function do not lead to change of its shape, the Fokker-Planck equation can be reduced to an equation for the second order moments of the distribution function or Langevin equation in invariant or momentum space.

Three numerical algorithms are realized in BETACOOL in order to simulate the evolution of the ion distribution function: root mean square (rms) dynamics, the model beam (MB) algorithm, and the multiparticle tracking (MPT) algorithm [26,27]. Root mean square dynamics calculate the evolution over time of the rms parameters of the ion distribution function during motion in the storage ring under a common action of a few heating and cooling effects. Each effect can be artificially included or excluded from consideration in order to distinguish the influence of different processes. The rms algorithm is based on solving a system of four differential equations for second order momentum in all 3 degrees of freedom, plus one equation for beam intensity change:

$$
\begin{aligned}
\frac{d \varepsilon_{x}}{d t} & =\frac{\varepsilon_{x}}{\tau_{\text {hor }}} ; & \frac{d \varepsilon_{y}}{d t} & =\frac{\varepsilon_{y}}{\tau_{\text {vert }}} ; \\
\frac{d \varepsilon_{\text {long }}}{d t} & =\frac{\varepsilon_{\text {long }}}{\tau_{\text {long }}} ; & \frac{d N}{d t} & =\frac{N}{\tau_{\text {life }}} ;
\end{aligned}
$$

where $\varepsilon_{x, y, l}$ are rms emittance in one of transverse or longitudinal planes.

Characteristic times $\tau_{i}$ of the evolution of the beam parameters are estimated under the assumption of a Gaussian distribution function: 


$$
\frac{1}{\tau_{i}}=\frac{\varepsilon_{\mathrm{fin}}-\varepsilon_{\mathrm{in}}}{\varepsilon_{\mathrm{in}} T_{\mathrm{rev}}}
$$

where $T_{\text {rev }}$ is revolution time. Growth rates $1 / \tau_{i}$ are positive for heating processes while cooling rates are negative. The beam lifetime is inversely proportional to the average value of ion loss probability $\left\langle P_{\text {loss }}\right\rangle$ over revolution period $1 / \tau_{\text {life }}=-\left\langle P_{\text {loss }}\right\rangle / T_{\text {rev }}$.

An investigation into the ion beam dynamics for an arbitrary shape of the distribution function is performed using multiparticle (MB) simulations. Here, the ion beam is represented by an array of several thousand modeling particles, with the algorithm realizing a solution of the Langevin equation in invariant or momentum space. The heating and cooling processes lead to a change of the particle momentum components and number of particles. The MPT algorithm uses real particles with arbitrary distribution and modeling of optical elements based on a Hamiltonian with nonlinear and space charge terms. The molecular dynamic (MD) technique [41] is applied in BETACOOL as part of the MPT procedure with the assumption that particles in a coasting beam have a periodic distribution along the ring. For a bunched beam, one can simulate the beam dynamics using so-called "macroparticles." MD is used for simulating intrabeam scattering (IBS) as well as for studying crystalline beams. A detailed description of the BETACOOL procedures is contained in [27].

\section{B. Multiple scattering of ions and rms emittance growth}

In low-energy storage rings beam emittance growth is caused mainly by small-angle multiple Coulomb scattering of circulating ions on atoms and molecules of the residual gas (vacuum losses) as well as due to intrabeam scattering (IBS) when beam intensity is high. If an internal target is installed in the ring the circulating ions are lost because the projectile beam is scattered on the target atoms when particles cross the gas jet many times. The transverse beam emittance might also grow due to energy straggling (fluctuations of energy losses) if the dispersion is not zero in the ring.

In BETACOOL the target is treated equivalent to a thin lens in ion optics [42]. A collision of the incident ion with a thin target atom produces a small-angle kick which is of stochastic nature. After crossing the target the ion's coordinates do not change, but it does experience angular scattering, i.e., all three components of particle momentum are changed and the particle might be lost with some probability. A change of transverse momentum is related mainly to multiple Coulomb scattering of ions on the nuclei of target atoms. The longitudinal momentum of the ion is reduced due to ionization and excitation energy losses occur during the interaction of the ion with the electron shell of the target atom. Fluctuation of ionization energy losses leads to the growth of the beam momentum spread.
Introducing the mean square scattering angle per target traversal, $\theta_{\mathrm{rms}}^{2}$, one can describe the emittance change after $N$ target traversals [42]. In the presence of dispersion $D$ and the derivative of dispersion $D^{\prime}$, emittance growth depends not only on the rms scattering angle but also on the momentum spread introduced by fluctuations of the ionization and excitation energy losses:

$$
\begin{aligned}
\Delta \varepsilon_{h, v}= & \varepsilon-\varepsilon_{0} \\
= & N \frac{\beta_{h, v} \theta_{\mathrm{rms}}^{2}}{2}+\frac{N}{2}\left(\frac{\left(1+\alpha_{h, v}\right)^{2}}{\beta_{h, v}} D_{h, v}^{2}\right. \\
& \left.+2 \alpha_{h, v} D_{h, v} D_{h, v}^{\prime}+\beta_{h, v} D_{h, v}^{\prime}{ }^{2}\right) \delta_{\mathrm{rms}}^{2},
\end{aligned}
$$

where $\alpha, \beta, \gamma$ are the Twiss parameters and $\delta_{\mathrm{rms}}^{2}$ is the mean square relative momentum deviation per target traversal. In order to reduce the growth rate of beam emittance, maximize the luminosity of the experiment, and improve the lifetime of ions, one should therefore provide low-beta mode and achromatic conditions at the target location.

Small-angle Coulomb scattering is well studied at intermediate and high energies and can be obtained from the Rutherford cross section [43]. As no specific expressions for ion scattering at an ultra-low-energy range exist which are of interest to our studies, we therefore apply the expression for mean square scattering angle to each scattering event based on Moliere's formula for a screened ion-atom potential [44] of the Thomas-Fermi type [45] to Fano's contribution of ion inelastic collision with atomic electrons [46],

$$
\theta_{\mathrm{str}}^{2}=2 \pi \rho x\left(\frac{Z_{T} Z_{i} r_{p}}{A_{i} \beta^{2} \gamma}\right)^{2}\left[\ln \left(\frac{\alpha_{2}^{2}}{\chi^{2}}\right)-1+\Delta b\right] .
$$

Here $\rho$ and $x$ are target density and thickness. $A_{T}, A_{i}, Z_{T}$, and $Z_{i}$ are the mass number and charge of the target and projectile ion, $\beta, \gamma$ are the relativistic factors, and $r_{p}$ is the classical proton radius. The parameter $\alpha_{2}$ is the upper integration constant of the rms scattering angle and is proportional to the ratio of the De Broglie wavelength and the sum of the target and projectile nuclei radii. The screening angle $\chi$ takes into account the deviation from the Born approximation [47]. The inelastic collision factor with atomic electrons $\Delta b$ was introduced by Fano [48] and includes a constant determined by the electron configuration of the target atom and estimated from the Thomas-Fermi model.

When the beam crosses the target, ion energy is lost due to excitation and ionization of atomic electrons. The expectation of the mean energy loss $\Delta E_{\mathrm{BB}}$ for one target traversal is calculated in accordance with the simplified Bethe-Bloch equation [49]. Fluctuations in energy loss might be estimated by the square of the standard deviation of the ion distribution function in energy space. The mean energy loss leads to deceleration of the beam while the 
fluctuation of the energy loss causes growth in ion momentum spread.

Growth rates due to the scattering of ions on the residual gas are calculated by the same methods as for a thin internal target such as a gas jet. The residual gas model is composed of gas cell targets which are distributed along the whole circumference of the ring. The rest gas heating rates are integrated over the whole lattice structure and lattice functions are used from each optical element. The effective partial densities of the residual gas components are calculated from the input vacuum value and the percentage of gas composition, and energy losses are estimated with the Bethe-Bloch formulas. Finally, the characteristic growth rates $1 / \tau_{i}$ are derived in accordance with the percentage of gas components.

The ion loss probability after crossing the target is proportional to the linear target density $\rho \cdot x$. The total cross section of ion losses is a sum of the cross sections of the different processes that lead to particle losses. Here the most significant factors are single scattering on large angles $\sigma_{\mathrm{ss}}$, the capture of an electron or the charge-exchange $\sigma_{\mathrm{ec}}$ in general, and nuclear reactions in the target at high energies $\sigma_{\mathrm{nr}}$. The cross section of single scattering at an angle larger than the acceptance angle $\theta_{\text {acc }}$ of the ring might be estimated by the formula

$$
\sigma_{\mathrm{ss}}=4 \pi\left(\frac{Z_{T} Z_{p} r_{p}}{A \gamma \beta^{2}}\right)^{2} \frac{1}{\theta_{\mathrm{acc}}^{2}}
$$

where $r_{p}$ is the classical proton radius. The acceptance angle is estimated by the expression

$$
\theta_{\mathrm{acc}}^{2}=\frac{A_{x, y}}{\pi \beta_{x, y}}\left(1-\frac{\varepsilon_{x, y}^{2}}{A_{x, y}^{2}}\right)
$$

where $A_{x, y}$ is the ring acceptance at the $x$ or $y$ plane and $\beta_{x, y}$ is the corresponding value of the betatron function at the target position. Similar expressions are used to estimate beam losses due to multiple scattering of ions on the residual gas.
Intrabeam scattering (IBS) refers to the small-angle Coulomb scattering of ions in the beam, and IBS effects in circular accelerators are of particular interest here. IBS is essentially a diffusion process and leads to the relaxation of ions at thermal equilibrium and to diffusion growth of beam volume in $6 \mathrm{D}$ phase space. This is due to the variation of lattice parameters along the ring circumference, as well as the presence of dispersion in the ring and coupling between longitudinal and transverse motion. Analytical calculations of IBS growth rates are based on the estimation of particle momentum variation caused by Coulomb interaction with other ions in the beam. The MD technique is also applied to a coasting beam [26]. For the IBS process, both techniques represent each optical element of the ring as a map, and the IBS growth rate is then averaged over all elements of the ring. The models of Piwinski [50], Martini [51], Bjorken-Mtingwa [52], and Jie-Wei [53] are some of the IBS scattering models that can be chosen in BETACOOL. The smooth focusing approximation IBS model of Piwinski neglects the betatron function derivative $\beta^{\prime}$ and dispersion function derivative $D^{\prime}$ and does not require a lattice structure, but does require mean values of the lattice functions. The Bjorken-Mtingwa model is based on a quantum-mechanical approach, and both the Martini and Bjorken-Mtingwa models account for the ring structure, i.e., the variation of beta functions in a ring lattice.

\section{BENCHMARKING OF ELISA EXPERIMENTS}

The long-term beam dynamics during beam circulation in ELISA have been investigated using BETACOOL. To benchmark our simulations we chose the same beam parameters as in the ELISA tests with coasting $\mathrm{O}^{-}$and $\mathrm{Mg}^{+}$ ions, see Table II. Small-angle multiple scattering of the circulating ions on the residual gas atoms and molecules, IBS, energy straggling, and charge exchange were all included in our simulations. We assumed that ions in the ELISA ring are lost on the elements of ring aperture because of the high growth rate of beam emittance and

TABLE II. BETACOOL beam parameters of the ELISA ring.

\begin{tabular}{lcc}
\hline \hline Ion & 16 & ${ }^{24} \mathrm{Mg}$ \\
Charge & -1 & +1 \\
Ion energy, keV & 22 & 18.4 \\
Initial beam intensities & $5 \times 10^{5}-1.6 \times 10^{7}$ & $2.7 \times 10^{7}$ \\
Ring circumference, m & 7.616 & 7.616 \\
Initial horizontal/vertical rms emittance, $\pi$ mm mrad $(\sigma)$ & $1 / 1$ & $0.7 / 0.35$ \\
Ring acceptance ESD-CYL, $\pi$ mm mrad & 10 & 10 \\
Ring acceptance ESD-SPH, $\pi$ mm mrad & 6 & 6 \\
Initial rms momentum spread, $\Delta P / P$ & $10^{-3}$ & $10^{-4}$ \\
Equilibrium rms momentum spread, $\Delta P / P$ & $\sim 4 \times 10^{-3}$ & $\sim 1.5 \times 10^{-3}$ \\
Vacuum pressure (hydrogen), Torr & $2 \times 10^{-11}$ & $10^{-11}$ \\
Electron detachment lifetime of $\mathrm{O}^{-}, \mathrm{sec}$ & 26 & \\
Lifetime of $\mathrm{O}^{-}$at $22 \mathrm{keV}, \mathrm{sec}$ & $\sim 12$ & \\
\hline \hline
\end{tabular}


the low ring acceptance. It was found that the reduction in beam intensity due to single scattering on large angles at a vacuum level of $10^{-11}$ Torr is essentially less than that caused by other factors, even at such a low beam energy, and we have therefore excluded the process of single scattering from further consideration.

The lifetime of $\mathrm{O}^{-}$ions due to electron detachment during the process of ion collision with the residual gas has been measured [15] and a value $\tau_{\exp }=26 \mathrm{~s}$ was used as a BETACOOL input parameter. In our studies we assumed a low ring acceptance even with cylinder deflectors, $A_{\mathrm{CYL}} \sim 10 \pi \mathrm{mm}$ mrad. On this basis, the measured rates of beam intensity decay for a $22 \mathrm{keV} \mathrm{O}^{-}$beam have been reproduced with good accuracy, see Fig. 2(b) and compare to Fig. 2(a). This gave rise to the conclusion that the main reason for beam size growth in electrostatic storage rings is multiple scattering of ions on the atoms and molecules of the residual gas, i.e., vacuum losses as well as Coulomb repulsion of the ions from each other at high intensities, i.e., IBS. As a consequence of fast beam size growth, the ions are then lost on the ring aperture because of a rather small ring acceptance. The rate of beam loss increases at higher intensities because IBS adds to vacuum loss. The initial shape of the decay curve depends on the emittance, the momentum spread, and the intensity of the injected beam.

The kinetics of negative oxygen ions have been studied at different initial intensities, from $5 \times 10^{5}$ up to $1.6 \times 10^{7}$ particles in the beam. The IBS effect is clearly seen in Fig. 2 as an excessive drop of beam current during the first few seconds when the beam intensity is still high (the green and red curves correspond initially to $8 \times 10^{6}$ and $1.6 \times$ $10^{7}$ ions, respectively). The long-term slope of this loss curve is determined by the ring acceptance and by the rate of multiple scattering on the residual gas, i.e., to the pressure level in the ring. The slope of the decay curve also depends on the lifetime due to electron detachment for negative ions or electron stripping for positive ions. At very low beam intensities, when IBS is negligible and only multiple scattering on the residual gas is present, the loss rate does not depend on the beam current or details of the ring lattice. For this reason, all lines are almost parallel towards the end of the cycle.

An exception is seen in the pink curve with more tilted slope, representing the decay of the beam intensity in ELISA with spherical deflectors. Our conclusion is that significantly higher loss rates are caused in this case by the reduced ring acceptance. Also, the effect of IBS is stronger when the beam density is high, and therefore particularly in regions where the beam is strongly focused. The IBS rates for ELISA with spherical deflectors are higher than with cylindrical electrodes because of the double focusing effect of the former and the resulting small beam size in both planes. As highlighted in the previous section of this paper, the ELISA ring acceptance in the vertical plane is mainly restricted by the sextupole component of the electric field of electrostatic deflectors and should be less than $30 \pi \mathrm{mm}$ mrad. Any misalignments of the beam equilibrium orbit out of the central curve in the space between the deflector plates will further reduce the area available for the stable motion. Thus, the real value of ring acceptance will be even less than what followed from our idealized OPERA-3D model studies.

Since the long-range slope of the decay curve depends on the ring acceptance and is in full agreement with the fact that the ring acceptance with spherical electrodes is smaller than with cylindrical electrodes, the fast decay in ELISA with spherical deflectors can be explained by the small acceptance $A_{\text {sph }} \approx 6 \pi \mathrm{mm}$ mrad.

We have compared the measured equilibrium profile of an $18.4 \mathrm{keV} \mathrm{Mg}^{+}$beam in ELISA with deflectors of spherical shape to BETACOOL simulations made under the assumption that the transverse beam size is mainly defined by losses on elements of the ring structure, see Fig. 4 . The simulated rms width $\sigma=1.5 \mathrm{~mm}$ corresponds to a ring acceptance of $A \sim 6 \pi \mathrm{mm} \mathrm{mrad}$, see Fig. 4(a). These results are in good agreement with the ELISA experiments where a beam profile of full width on half maximum

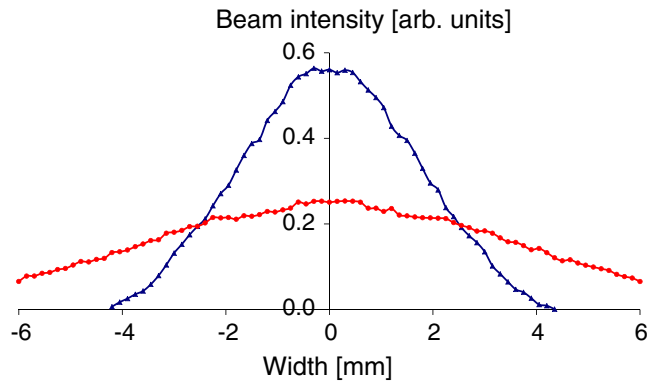

(a)

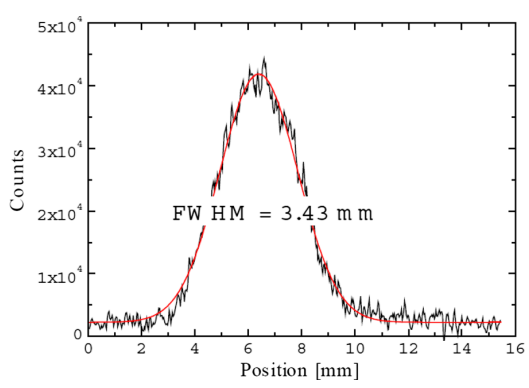

(b)

FIG. 4. Beam profile of $18.4 \mathrm{keV} \mathrm{Mg}^{+}$ions in the ELISA ring with spherical deflectors: (a) BETACOOL simulations. The blue curve corresponds to a ring acceptance of $A=6 \pi \mathrm{mm} \operatorname{mrad}(\sigma=1.5 \pi \mathrm{mm} \mathrm{mrad})$ while the red curve is a predicted beam profile assuming a ring acceptance of $50 \pi \mathrm{mm} \mathrm{mrad}$; (b) measured profile, corresponding to a ring acceptance of about $5 \pi \mathrm{mm} \mathrm{mrad}\left(\beta_{x}=3.5 \mathrm{~m}\right)$, plot taken from [15]. 


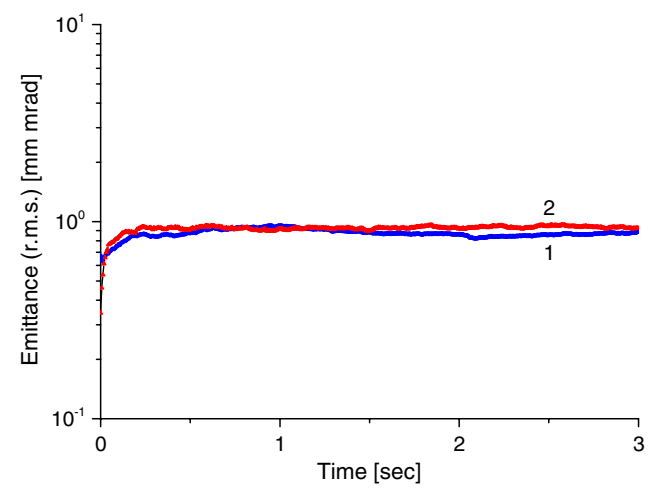

(a)

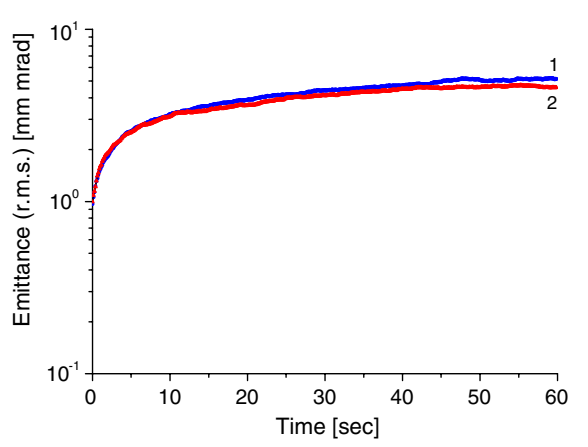

(b)

FIG. 5. Evolution of rms emittance in ELISA in horizontal (blue) and vertical (red) planes: (a) $\mathrm{Mg}^{+}$beam grows to the ring acceptance of $A=6 \pi \mathrm{mm}$ mrad in the first $200 \mathrm{~ms}$ after injection, such fast growth was observed in ELISA; (b) assuming a ring acceptance as large as $\boldsymbol{A}=50 \pi \mathrm{mm}$ mrad the emittance of the $\mathrm{O}^{-}$beam should grow steadily in $\sim 50 \mathrm{~s}$ which contradicts ELISA measurements.

$\mathrm{FWHM}=3.43 \mathrm{~mm}$ was measured at the point where the horizontal beta function is equal to $\beta_{x}=3.5 \mathrm{~m}$ [Fig. 4(b)]. The ring acceptance was then varied as an input parameter in BETACOOL. Assuming a ring acceptance of $A \approx$ $50 \pi \mathrm{mm} \mathrm{mrad}$, the resulting $\mathrm{rms}$ beam size was $\sigma \approx$ $3 \mathrm{~mm}$, i.e., twice that measured in the experiment, see Fig. 4(a).

The beam size of $\mathrm{Mg}^{+}$ions was measured as a function of time, and no increase in the width was seen over the first $0.3 \mathrm{~s}$ [15]. We explain the steady beam size in the ELISA experiments by the fact that the initially high-intensity $\mathrm{Mg}^{+}$beam $\left(N_{0}=2.7 \times 10^{7}\right.$ ions $)$ spreads out to the ring aperture due to a small ring acceptance in the first $\sim 200 \mathrm{~ms}$ after injection, see Fig. 5(a), and no further increase of beam emittance occurs, only beam losses on ring elements. In contrast, for a ring acceptance as large as

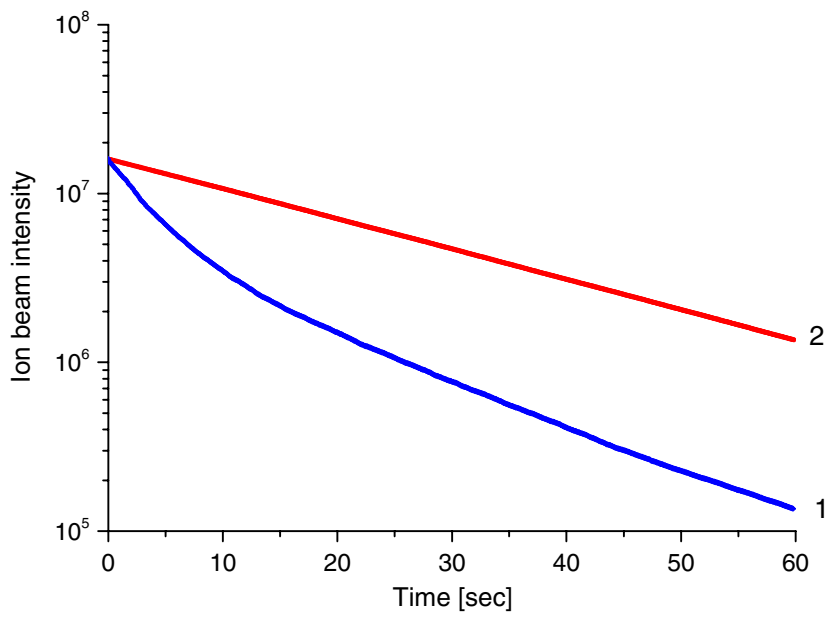

FIG. 6. BETACOOL simulations of beam loss rates in ELISA. The rate of intensity decay in the ring with acceptance $A=$ $10 \pi \mathrm{mm}$ mrad corresponds to a lifetime $\tau=12 \mathrm{~s}$ (blue curve 1 ). Assuming a ring acceptance of $A=50 \pi \mathrm{mm}$ mrad the lifetime would be $\tau=24$ s (red curve 2), i.e., double the observed value.
$A=50 \pi \mathrm{mm}$ mrad, the beam emittance in ELISA should grow steadily for at least 50 seconds during the circulation of $\mathrm{O}^{-}$ions in the ring, see Fig. 5(b), which is not in agreement with experimental data.

We have included all listed heating processes in the BETACOOL model, as well as the experimentally defined lifetime due to detachment of negative oxygen. The lifetime of an $\mathrm{O}^{-}$beam circulating in the ELISA ring, estimated by counting the beam intensity decay rate, is $\tau_{1 / 2} \approx 12 \mathrm{~s}$, see blue curve in Fig. 6. However, for an acceptance as large as $A=50 \pi \mathrm{mm}$ mrad the lifetime of the $\mathrm{O}^{-}$beam should be at least $24 \mathrm{~s}$ (red curve), which contradicts the measured value of $\tau_{1 / 2} \approx 12 \mathrm{~s}$.

Because of fluctuations in ion energy loss on molecules of the residual gas (i.e. energy straggling), the initial

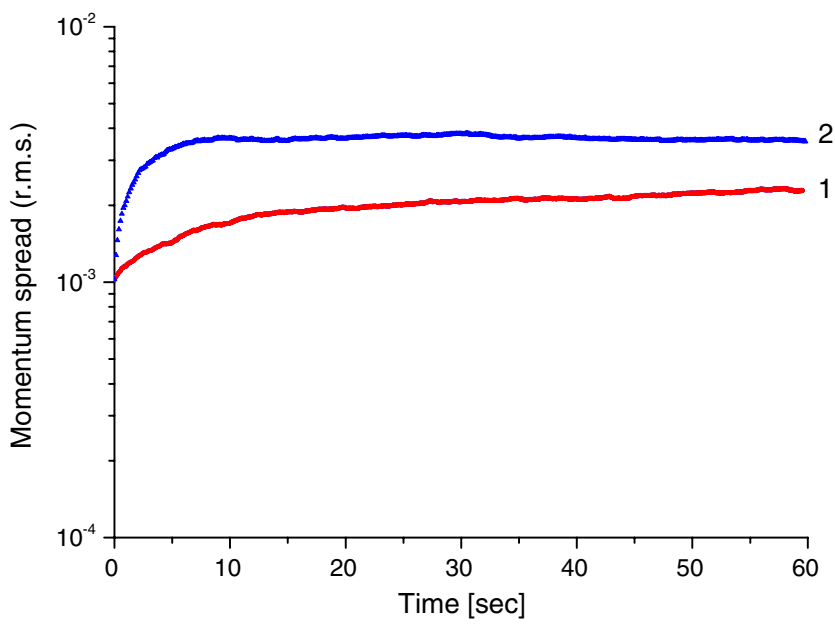

FIG. 7. Evolution of rms momentum spread of a $22 \mathrm{keV} \mathrm{O}^{-}$ beam. While $10^{6}$ ions are injected in the ring (red curve 1), equilibrium is $d p / p \approx 2.2 \times 10^{-3}$ (rms). When $1.6 \times 10^{7}$ ions are injected in the ring (blue curve 2), the growth rates are higher and equilibrium momentum spread is $d p / p \approx 4 \times 10^{-3}$ (rms). 


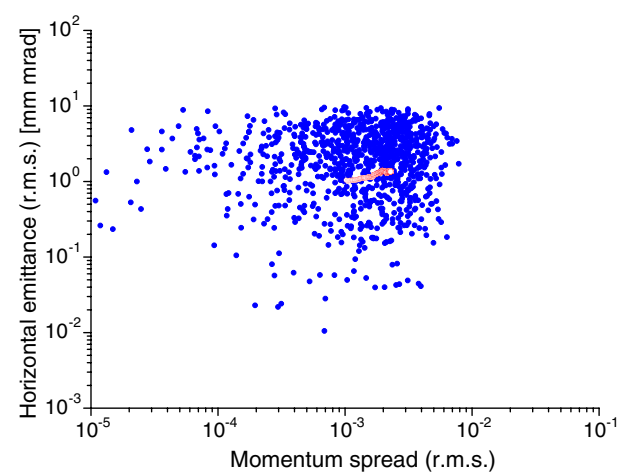

(a)

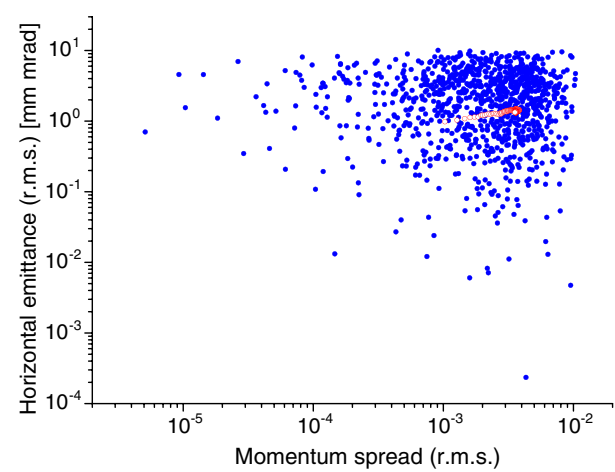

(b)

FIG. 8. Distribution of a $22 \mathrm{keV} \mathrm{O}^{-}$ion beam in 3D phase space $\left(\Delta p / p, \varepsilon_{x}\right)$. Scattered points (blue) are particle invariants simulated in the frame of the model beam algorithm. Evolution of rms emittance and momentum spread in time is outlined by red circled points: (a) low-intensity beam $\left(N_{o}=10^{6}\right.$ ions), the beam distribution in energy is of Gaussian shape; (b) high-intensity beam $\left(N_{o}=\right.$ $1.6 \times 10^{7}$ ions), the ions with high momentum spread are cut off by longitudinal ring acceptance.

momentum spread of the oxygen beam increases until equilibrium conditions are reached. At initial low beam intensity $\left(N_{0}=10^{6}\right.$ ions) the rms momentum spread doubles from $10^{-3}$ to $\sim 2 \times 10^{-3}$, see red curve in Fig. 7, while at high intensities $\left(N_{0}=1.6 \times 10^{7}\right.$ ions $)$ the effect of intrabeam scattering in the longitudinal phase space is more pronounced, and here momentum spread quadruples to $\sim 4 \times 10^{-3}$ and reaches ring acceptance, see blue curve in Fig. 7.

The behavior of a $22 \mathrm{keV} \mathrm{O}^{-}$ion beam in 3D phase space is shown in Fig. 8 where the distribution of the beam emittance is plotted against the distribution of the beam momentum spread. Ions are injected into the ring with an initial emittance of $\varepsilon_{x}=1 \pi \mathrm{mm} \mathrm{mrad}(\mathrm{rms})$ and initial momentum spread of $d p / p=10^{-3}$ (rms). We compared the 3D phase space distribution at both low and high initial beam intensities. Because of the high growth rate of transverse emittance, the beam reaches the ring aperture in the first few seconds even with a low injected beam current. That is why the beam distribution in the transverse direction is sharply cut off by the ring acceptance at $A_{x}=$ $10 \pi \mathrm{mm}$ mrad. The growth rate of momentum spread due to the fluctuation of energy losses depends on the initial beam intensity. We determined special conditions which allowed us to couple the transverse motion invariant with the longitudinal momentum spread of a coasting beam through the BETACOOL model beam algorithm. The ion is then considered to be lost as soon as the momentum spread exceeds the limit defined by the following expression:

$$
\operatorname{Inv}_{\text {long }} \cdot\left\langle D_{x}\right\rangle^{2}>\operatorname{accept}_{x} \cdot\left\langle\beta_{x}\right\rangle
$$

Here, $\left\langle D_{x}\right\rangle$ and $\left\langle\beta_{x}\right\rangle$ are average values of dispersion and horizontal betatron function over the ring circumference, and accept $t_{x}$ is a predefined ring acceptance. The current value of the longitudinal motion invariant of the coasting beam calculated for each model particle is twice the longitudinal rms emittance $\operatorname{Inv}_{\text {long }}=2 \varepsilon_{\text {long }}^{\mathrm{rms}}=2\left(\Delta p^{\mathrm{rms}} / p\right)^{2}$.
We applied these conditions to a circulating beam of $\mathrm{O}^{-}$ ions in ELISA and found that the momentum spread of low-intensity ( $N_{0}=10^{6}$ ions) beam is less than the longitudinal ring acceptance and that the momentum

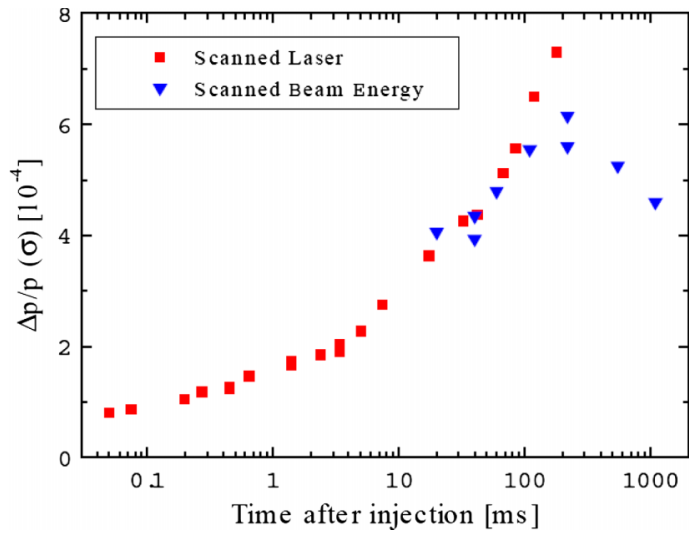

(a)

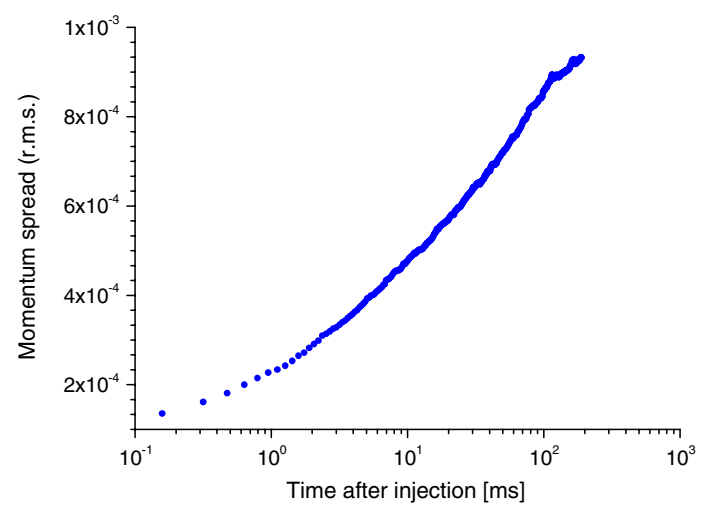

(b)

FIG. 9. Momentum spread of an $18.4 \mathrm{keV} / \mathrm{A} \mathrm{Mg}^{+}$beam as a function of time. The initial beam intensity is $2.7 \times 10^{+7}$ ions: (a) experimental data taken from [14]; (b) BETACOOL simulations. Because of IBS the rms velocity spread increases from around $d p / p=10^{-4}$ at injection to $d p / p=7 \times 10^{-4}$ in about $200 \mu \mathrm{s}$. 
distribution for small currents is Gaussian, see Fig. 8(a). At high initial beam intensities IBS adds to the energy spread growth which increases to the limit where the distribution is sharply cut off at $\Delta p / p=1 \%$, see Fig. 8(b). Thus, we can conclude that the energy acceptance of the ELISA ring is about $\Delta E / E=2 \%$.

In addition, the fast growth in momentum spread of a high-intensity $\left(2.7 \times 10^{7}\right.$ ions $)$, low-energy $(18.3 \mathrm{keV})$ $\mathrm{Mg}^{+}$beam has been reproduced. For example, a "blow up" in rms momentum spread from around $10^{-4}$ at beam injection to $7 \times 10^{-4}$ about $150 \mathrm{~ms}$ after injection has been measured in the ELISA ring, see Fig. 9(a), and our simulations have achieved excellent agreement with these experimental results [Fig. 9(b)], demonstrating that a fast increase in momentum spread during the first moments after beam injection is mainly caused by intrabeam scattering in longitudinal phase space. Results from measurements that a high-intensity beam with a small initial momentum spread of $\Delta p / p=10^{-4}$ grows more or less instantaneously to $\Delta p / p=10^{-3}$ were confirmed in our simulations.

\section{OPERATION WITH INTERNAL TARGET}

Based on the results on the previous sections, we have studied the kinetics of ultra-low-energy antiprotons circulating in a small recycler storage ring and their interaction with an internal supersonic gas jet target. This recycler ring is made of electrostatic elements and has been designed by the QUASAR Group [54] in close collaboration with the ASACUSA-MUSASHI collaboration and colleagues from the Max Planck Institute for Nuclear Physics in Heidelberg [55].

The measurement of ionization cross sections in collisions between low-energy beams of antiprotons and different target gases, such as for example helium, directly addresses the few-body Coulomb problem and provides experimental data to aid in understanding many-body interactions. Studying ionization by antiproton impact rather than electron and proton impact is an advantage because competing processes such as exchange and/or capture of bound electrons are excluded during antiproton impact, while the multiple scattering of antiprotons on the nuclei of target atoms might be distinguished from ionization measurements by a coincidence scheme in the experimental setup of the reaction microscope [56].

In order to understand the ionization interaction processes, it is necessary to obtain fully differential cross sections in experiments where the momentum vectors of the He ions and electrons from the ionization events by antiproton impact can be measured. So far, low-energy antiproton experimental studies have been limited to the level of total cross sections due to the single pass setup of the experiments and the very limited availability of highquality low-energy antiproton beams. The advantage of incorporating the reaction microscope into the ring over the single pass experiment is that the antiproton beam can be recirculated and passed through the helium supersonic gas jet target for thousands if not millions of times, thus increasing the count rate to enable low-energy partial differential cross-section measurements.

Antiprotons circulate in the recycler with an energy of between 3 and $30 \mathrm{keV}$ and the ring has been specifically designed to provide a projectile beam size of $\sim 1 \mathrm{~mm}$ and a divergence angle of no more than $20 \mathrm{mrad}$ in the center of a dedicated reaction chamber. The ring layout and lattice parameters are presented elsewhere [55]. The simple racetrack lattice with two straight sections each $1.3 \mathrm{~m}$ long has been optimized for studies requiring multiple interactions between the ions of the circulating beam and atoms of a helium gas jet target at the crossing point where the Reaction Microscope will be located. Two sets of lowbeta inserts located in both straight sections are needed to provide a sharp focus and reduced beta functions in the interaction region to $\beta_{x, y} \leq 10 \mathrm{~cm}$. The dispersion function is vanished in long straight sections to achromatic conditions, $D=D^{\prime}=0$. A combination of low-beta mode and achromatic conditions allows the count rate of ionization events to be increased by operating the reaction microscope with a high-density gas jet target of up to $n_{\text {tgt }}=10^{12} \mathrm{~cm}^{-3}$.

The long-term beam dynamics in the recycler ring were studied. In addition to multiple scattering of the circulating antiprotons on the residual gas, IBS and beam losses on the ring aperture, we also investigated into small-angle multiple scattering of ions on the nuclei of the helium gas jet target. The effects of mean energy loss and fluctuations of energy loss due to excitation and ionization of the electron shell of the helium atoms by the incident beam were also included.

We compared the ring performance at different initial conditions: low $\left(N_{o}=5 \times 10^{5}\right)$ and high $\left(N_{o}=10^{7}\right)$ beam intensities, different rms emittance $\left(\varepsilon_{\mathrm{rms}}=1\right.$ and $2 \pi \mathrm{mmmrad}$ ), and different $\mathrm{rms}$ momentum spreads $\left(d p / p=10^{-3}\right.$ and $\left.10^{-4}\right)$. The feasibility of combined ring and target operation at 3 to $30 \mathrm{keV}$ beam energy range has been checked. Table III gives a summary of the ring, beam, and target parameters as well as results of numerical studies.

Similar to the ELISA studies, we assumed that due to the multiple scattering of ions on the residual gas and target atoms, as well as due to IBS at high beam intensities, the ultra-low-energy antiproton beam is spread out and lost on the ring aperture because of the limited acceptance of the recycler ring estimated to be $A_{y}=15 \pi \mathrm{mm} \mathrm{mrad} \mathrm{[55].}$ The growth of the beam emittance and momentum spread at $3 \mathrm{keV}$ is much faster than at $30 \mathrm{keV}$. But in the considered energy range the equilibrium rms beam emittance is limited by the ring acceptance regardless of ion energy, initial intensity, or initial emittance of the injected beam. Providing achromatic conditions are realized the 
TABLE III. Overview of the recycler ring parameters

Ring circumference, $\mathrm{m}$

Betatron tunes $\nu_{x} / \nu_{y}$

Length of straight section/available for experiments, $\mathrm{mm}$

Ring acceptance, $\pi$ mm mrad

Vacuum pressure (hydrogen), Torr

Horizontal/vertical beta functions at target $\beta_{X} / \beta_{Y}, \mathrm{~m}$

Dispersion and derivative of dispersion at target, $\mathrm{m}$

Full size of beam spot at target location, $\boldsymbol{X} \times \boldsymbol{Y}, \mathrm{mm}$

Processes included in simulations

IBS models

Helium target diameter, $\mathrm{mm}$

Helium target density, $\mathrm{cm}^{-3}$

Equilibrium momentum spread

Antiproton energy, keV

Rotation period of antiprotons in the ring, $\mu \mathrm{s}$

Ionization cross section of $\mathrm{He}$ atoms by antiprotons, $\mathrm{cm}^{-2}$

Initial intensity of injected ions, $N_{0}$

Initial rms emittance, $\pi \mathrm{mm}$ mrad ( $1 \sigma$ emittance)

Initial rms momentum spread, $d p / p$

Beam lifetime $\tau, \mathrm{ms}$

Time to achieve $90 \%$ of all ionization events, ms

Integral of ionization events

Average rate of events per revolution in the ring

\begin{tabular}{|c|c|c|c|}
\hline \multicolumn{4}{|l|}{8.165} \\
\hline \multicolumn{4}{|l|}{$3.315 / 1.76$} \\
\hline \multicolumn{4}{|l|}{$1300 / 466$} \\
\hline \multicolumn{4}{|l|}{$15 \pi$} \\
\hline \multicolumn{4}{|l|}{$10^{-11}$} \\
\hline \multicolumn{4}{|l|}{$0.1 / 0.1$} \\
\hline \multicolumn{4}{|c|}{$D=D^{\prime}=0$} \\
\hline \multicolumn{4}{|c|}{$2 \times 2$} \\
\hline \multicolumn{4}{|c|}{ rest gas + gas jet target + IBS + acceptance } \\
\hline \multicolumn{4}{|c|}{ Martini and Bjorken-Mtingwa Gaussian } \\
\hline \multicolumn{4}{|c|}{1} \\
\hline \multicolumn{4}{|l|}{$5 \times 10^{11}$} \\
\hline \multicolumn{4}{|c|}{$\sim 8 \times 10^{-3}$} \\
\hline 3 & & 30 & \\
\hline 10.7 & & 3.4 & \\
\hline $2 \times 10^{-17}$ & & $5 \times 10^{-17}$ & \\
\hline $5 \times 10^{5}$ & $10^{7}$ & $5 \times 10^{5}$ & $10^{7}$ \\
\hline 2 & 1 & 2 & 1 \\
\hline $10^{-3}$ & $10^{-4}$ & $10^{-3}$ & $10^{-4}$ \\
\hline 130 & 100 & $\sim 1500$ & $\sim 1300$ \\
\hline 300 & 200 & $\sim 2.5 \mathrm{~s}$ & $\sim 1.5 \mathrm{~s}$ \\
\hline $3.9 \times 10^{3}$ & $2.6 \times 10^{4}$ & $3.5 \times 10^{5}$ & $3.2 \times 10^{6}$ \\
\hline$\sim 0.12$ & $\sim 1.2$ & $\sim 0.5$ & $\sim 7$ \\
\hline
\end{tabular}

$15 \pi$

$D=D^{\prime}=0$

$2 \times 2$

rest gas + gas jet target + IBS + acceptance

Martini and Bjorken-Mtingwa Gaussian

1

$5 \times 10^{1}$

$\sim 8 \times 10^{-3}$

10.7

$2 \times 10^{-17}$

$5 \times 10^{5}$

$10^{-3}$

$3.9 \times 10^{3}$

$\sim 1.2$
1

$\sim 1.5 \mathrm{~s}$

$\sim 7$ equilibrium beam spot at the target depends on ring acceptance and the value of the betatron functions $\beta_{y}^{\text {tgt }}$ at the target location $y=\sqrt{\beta_{y}^{\text {tgt }} \cdot A_{y}}$. With a low-beta achromatic lattice and a rather small ring acceptance, the beam size at the target can be reduced to $2 \mathrm{~mm}$ in diameter. In the focus point the angular divergence is less than the $10 \mathrm{mrad}$, as required by the experiment. The beam shape at the target location might be adjusted by variation of the horizontal and vertical betatron functions. Nevertheless, due to nonlinear astigmatic aberrations it would be difficult to reduce the beam size further to $\sim 1 \mathrm{~mm}$. A combination of a reduced ring acceptance (beam collimation) and very small betatron functions at the location of the target might help achieving a $1 \mathrm{~mm}$ beam spot on the target, but at the expense of an increased loss rate.

Growth of the beam momentum spread is caused by fluctuations of antiproton energy loss during the interaction of ions with the residual gas and internal gas jet target. Additional fluctuations of energy losses during multiple crossings of a high-density target $\left(n_{\mathrm{tgt}}=5 \times 10^{+11} \mathrm{~cm}^{-3}\right)$ will double the growth rate of energy loss fluctuations due to residual gas only and momentum spread reaches ring acceptance at $d p / p=8 \times 10^{-3}(\mathrm{rms})$. We compared beam distribution in $3 \mathrm{D}$ phase space of a $30 \mathrm{keV}$ highintensity $\left(N_{o}=10^{7}\right.$ ions) beam with an initial momentum spread of $d p / p=10^{-4}$ (rms), see Fig. 10(a), to the beam distribution of a $3 \mathrm{keV}$ low-intensity $\left(5 \times 10^{5}\right.$ ions $)$ beam with an initial momentum spread of $d p / p=10^{-3}$ (rms), see Fig. 10(b). Evolution of rms parameters is shown by red circled dots here and the tilted line presents equilibrium conditions. While growth rates are different at 3 and $30 \mathrm{keV}$ the equilibrium parameters are primarily determined by the ring acceptance and almost the same regardless of the initial conditions. Also, the distribution of the emittance as well as the distribution of the momentum spread (scattered points of particle invariants simulated in frame of the model beam algorithm) are sharply cut off at large values. Thus, it can be concluded that the antiproton beam fills the transverse ring acceptance $A_{x, y}=$ $15 \pi \mathrm{mm} \mathrm{mrad}$ as well as the longitudinal ring acceptance $\Delta p / p=2 \%$.

Furthermore, we studied mean energy loss of antiproton beams due to the excitation and ionization of atomic electrons of the gas jet target in accordance with the Bethe-Bloch formula [49]. The energy spread and mean energy loss of a high-intensity ( $N_{o}=10^{7}$ ions) antiproton beam interacting with a He target of $n_{\text {tgt }}=5 \times 10^{11} \mathrm{~cm}^{-3}$ density are shown in Fig. 11.

Antiproton ions injected into the ring at $3 \mathrm{keV}$ can lose almost $1.6 \%$ of initial beam energy in the first $200 \mathrm{~ms}$ of interaction with the internal target, see curve 1 in Fig. 11. At $30 \mathrm{keV}$ the rate of mean energy loss is a few times less and beam energy will be reduced to $0.6 \%$ of the initial value in the first $\sim 4 \mathrm{~s}$, see curve 2 in Fig. 11. Because of a lack of experimental data at these energies more detailed studies into energy loss are required. In our simulations we have assumed that the ratio between ionization and excitation events resembles the interaction between highenergy ions and target atoms. At $3 \mathrm{keV}$ more than $99.7 \%$ 


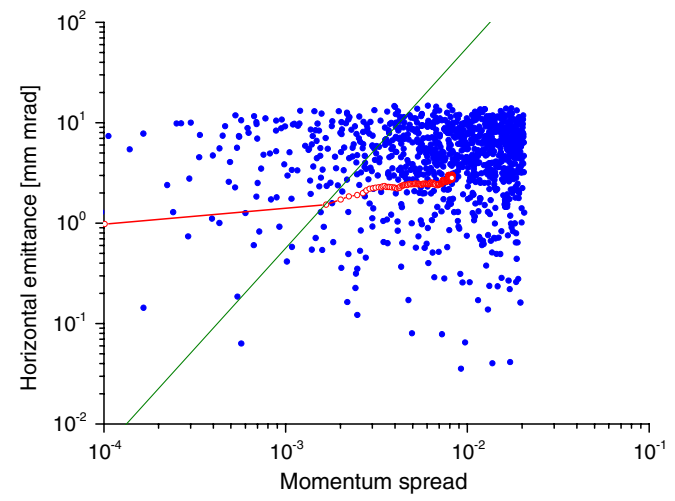

(a)

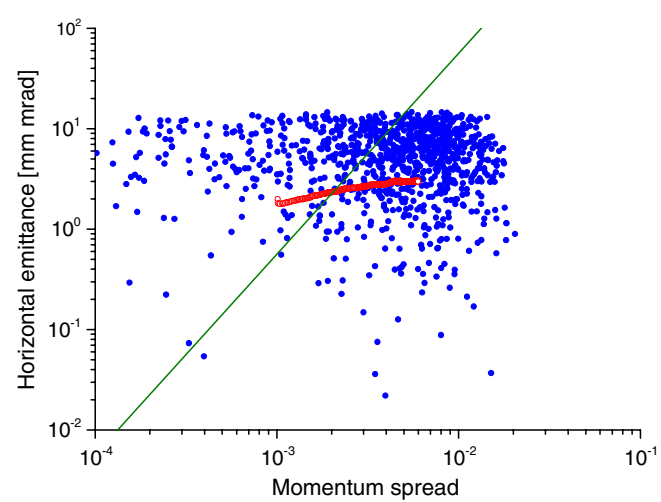

(b)

FIG. 10. Beam distribution in 3D phase space in the presence of a target. Scattered points (blue) are particle invariants simulated in the frame of the model beam algorithm. Evolution of rms parameters is shown by red circled dots and the tilted line presents equilibrium conditions: (a) $30 \mathrm{keV}$ high-intensity $\left(N_{o}=10^{7}\right.$ ions) beam with initial emittance $\varepsilon_{x}=1 \pi \mathrm{mm} \mathrm{mrad}$ (rms) and momentum spread $\Delta p / p=10^{-4}$ (rms); (b) $3 \mathrm{keV}$ low-intensity $\left(N_{o}=5 \times 10^{5}\right.$ ions) beam with initial emittance $\varepsilon_{x}=$ $2 \pi \mathrm{mm}$ mrad (rms) and momentum spread $\Delta p / p=10^{-3}$ (rms). While the growth rates are essentially different, the equilibrium parameters are restricted by the ring transverse as well as longitudinal acceptances and almost identical, regardless of the initial conditions.

of the ions will be lost due to multiple scattering on the target nuclei, and only $0.2 \%$ of incident beam will ionize $\mathrm{He}$ atoms. Thus, in our model the mean energy losses at $3 \mathrm{keV}$ result from excitation of the electron shell of $\mathrm{He}$ atoms, rather than by ionization. It should be pointed out that the results of the simulations are in better agreement with experimental ionization cross-section data at $30 \mathrm{keV}$ beam energy.

Even though the position of the beam will not be shifted when ions lose some of their energy in the target since achromatic conditions are applied in the long straight sections of the recycler ring, the energy losses should still be compensated, e.g., by means of a small drift tube rf system.

Beam intensity losses, lifetime, and ionization events due to antiproton impact with helium atoms were calcu-

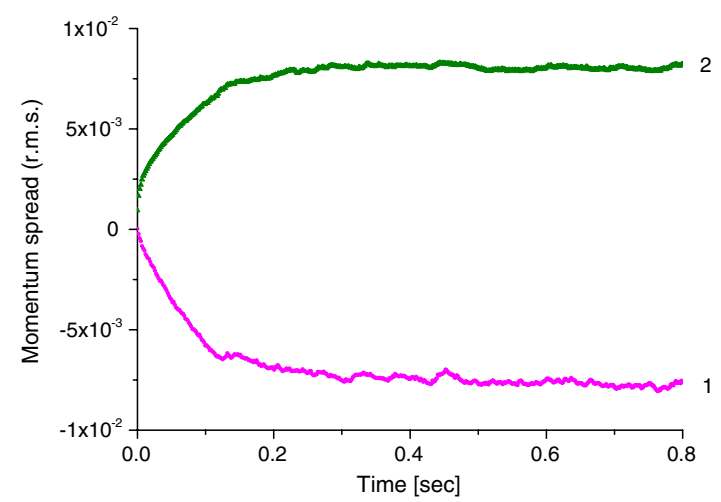

(a) lated for different target densities, for low- and highintensity beams, and at the lowest and highest beam energy, see Table III. Experimental data of the ionization cross section of He atoms by antiproton impact at different projectile energies [57] were used to estimate the number of useful events in the reaction microscope where the momentum vectors of the helium ions and electrons are measured. In the experimental data, it was assumed that every ionization event will lead to the loss of an antiproton. This assumption is not evident since the maximum transferable energy is less than $0.2 \%$ of the beam energy and thus lower than the longitudinal ring acceptance. If the ions continue to circulate in the ring after an impact and hence produce more than one ionization event, the count rate and total number of useful events will be higher than estimated here. At low beam

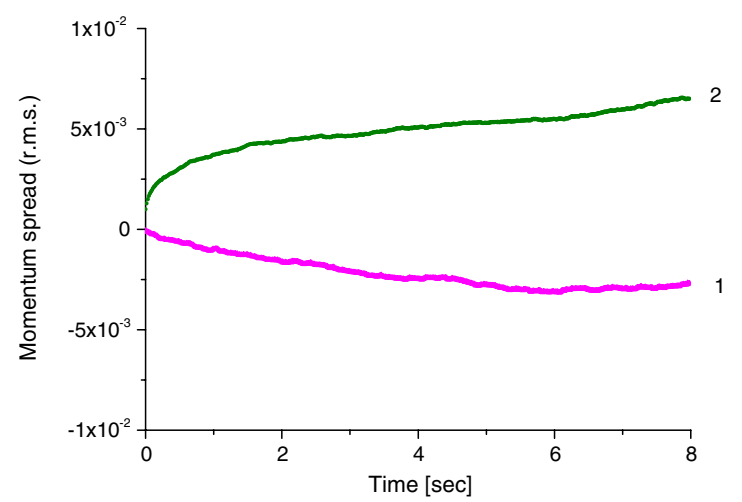

(b)

FIG. 11. Mean energy loss (pink curve 1) and energy straggling (green curve 2) of a high-intensity $\left(N_{o}=10^{7}\right.$ ions) antiproton beam interacting with a He target of $n_{\mathrm{tgt}}=5 \times 10^{+11} \mathrm{~cm}^{-3}$ density: (a) ion kinetic energy $3 \mathrm{keV}$, energy shift $\Delta T / T=-8 \times 10^{-3}$; (b) kinetic energy $30 \mathrm{keV}$, energy shift $\Delta T / T=-3 \times 10^{-3}$. 


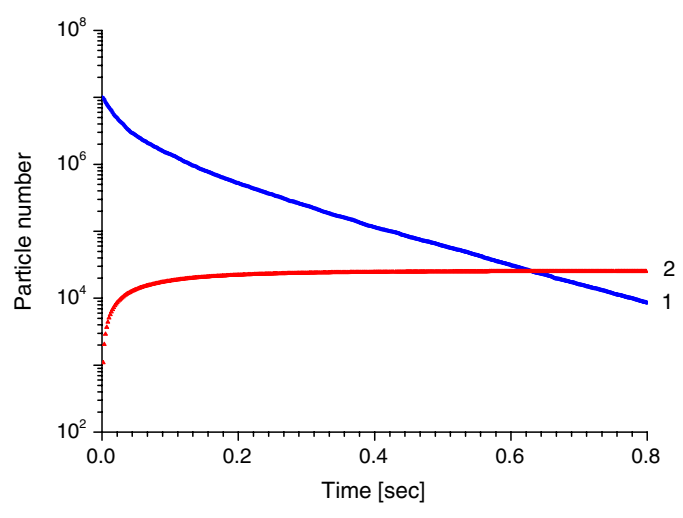

(a)

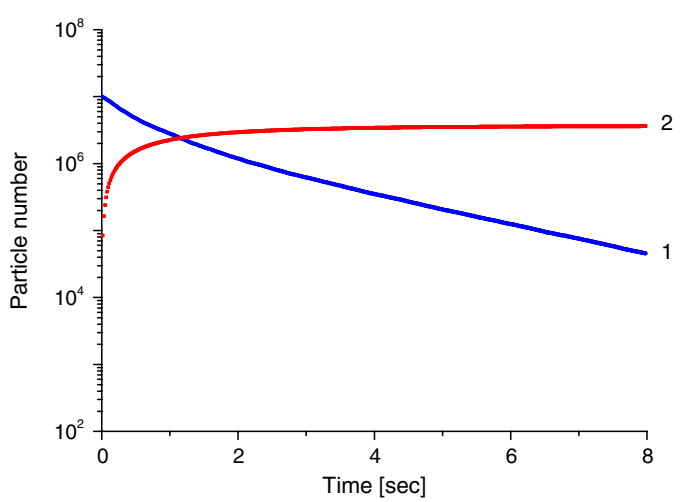

(b)

FIG. 12. Losses of a high-intensity ( $N_{o}=10^{7}$ ions) beam (black) and total number of ionization events (red) during multiple crossing of antiprotons and a helium gas jet. Target density is $n_{\mathrm{tgt}}=5 \times 10^{11} \mathrm{~cm}^{-3}$ : (a) energy of projectile ions is $3 \mathrm{keV}$, number of ionization events is $2.6 \times 10^{4}$ (curve 1), and lifetime is about $100 \mathrm{~ms}$ (curve 2); (b) energy of projectile ions is $30 \mathrm{keV}$, number of ionization events is $3.2 \times 10^{6}$ (curve 1 ), lifetime is $1.3 \mathrm{~s}$ (curve 2).

intensity, i.e. $N_{o}=5 \times 10^{5}$ ions, the average count rate in the ring is less than one event per revolution even at a high target density of $n_{\mathrm{tgt}}=5 \times 10^{11} \mathrm{~cm}^{-3}$, see Table III. Such a low rate is not very favorable for the operation of the reaction microscope.

Losses of an initially high intensity, $N_{o}=10^{7}$ ions beam (black) and the total number of ionization events (red) during multiple crossings of the stored antiprotons with a helium gas jet are shown in Fig. 12. The lifetime of a $3 \mathrm{keV}$ beam is limited to $\sim 120 \mathrm{~ms}$ because of the high multiple scattering rate of ultra-low-energy ions on a dense target, where $n_{\mathrm{tgt}}=5 \times 10^{11} \mathrm{~cm}^{-3}$. In total, over 24000 ionization events can be detected during the first $200 \mathrm{~ms}$ of beam circulation in the ring, see Fig. 12(a). Thereby, more than $99.7 \%$ of a $3 \mathrm{keV}$ beam will be lost on the ring aperture before the helium atoms can be ionized. At $3 \mathrm{keV}$ the beam will complete more than 20000 revolutions in the ring before it is completely lost with an average count rate of $\sim 1.2$ ionization events per revolution. This rate is reasonable for measurement of differential cross sections in experiments with a reaction microscope.

In the case of multiple crossings of a $30 \mathrm{keV}$ antiproton beam with $N_{o}=10^{7}$ ions and a high-density helium gas jet target, $n_{\text {tgt }}=5 \times 10^{11} \mathrm{~cm}^{-3}$, up to $3 \times 10^{6}$ ionization events might be detected in the first two seconds of ion circulation in the ring, see Fig. 12(b). The beam lifetime of $30 \mathrm{keV}$ antiprotons in the presence of a dense helium target is of the order of one second. In this case the average count rate is more than five events per turn. The beam lifetime of $30 \mathrm{keV}$ antiprotons circulating in the ring without a target is more than $5 \mathrm{~s}$. Thus, one may expect to accumulate a similar total number of ionization events even at a reduced gas jet density. In this case the count rate is less but since the gas load from the target is reduced the conditions for the experiment are more relaxed and beam lifetime increased.

\section{SIMULATIONS OF BEAM SHRINKING IN THE TSR ELECTRON COOLING EXPERIMENT WITH SLOW IONS}

Experiments with electron cooling of slow ions provide important information about the possible performance and limitations at the keV-energy range and are of crucial importance for future generation facilities, such as the CSR and the ultralow energy storage ring (USR). Cold electron beams at an energy range of $150 \mathrm{eV}$ down to $10 \mathrm{eV}$ and possibly even less are a key element of the next generation of electrostatic storage rings where electron cooling of $\mathrm{keV}$ ion beams needs to be achieved [58]. For this purpose, electron beams with a very low-energy spread at $\mathrm{eV}$ energies are required to guarantee lowest longitudinal beam temperatures and thereby increase the friction force of electron cooling.

\section{A. Application of a photocathode gun for electron cooling of low-energy ions}

Experiments with electron cooling of $\mathrm{CF}^{+}$ions $(A=31)$ at the Test Storage Ring (TSR) [59] of the Max Planck Institute for Nuclear Physics in Heidelberg, Germany have been reported [60], in which a low-intensity beam of $93 \mathrm{keV} / \mathrm{A} \mathrm{CF}^{+}$ions has been shrunk to an ultrasmall size using a cold beam of $53 \mathrm{eV}$ electrons produced by a cryogenically cooled GaAs photocathode.

$\mathrm{CF}^{+}$ions at an intensity of a few hundreds of $\mathrm{pA}$ have been injected into the TSR ring with an initial emittance of $\varepsilon_{x, y}^{\mathrm{in}} \approx 1 \pi \mathrm{mmmrad}$ and initial momentum spread of $(\Delta p / p)_{\text {in }}=2 \times 10^{-4}$. The TSR electron target setup was converted to act as an electron cooling device [61]. A cooling time of less than $\tau_{\text {cool }}<2 \mathrm{~s}$ and an equilibrium beam size of about $0.2 \times 0.04 \mathrm{~mm}^{2}(\mathrm{rms})$ after $\sim 6 \mathrm{~s}$ of cooler operation were measured. Assuming an upright orientation of the phase space ellipse in the middle of the straight section of the ring where the electron target is 
located, and subtracting $0.1 \mathrm{~mm}$ dispersion from the total $0.2 \mathrm{~mm}$ radial width of the beam, one may estimate the equilibrium emittance of cooled ions as $\varepsilon_{x, y}^{\mathrm{eq}} \sim(1-2) \times$ $10^{-3} \pi \mathrm{mm} \operatorname{mrad}(\mathrm{rms})$.

In this experiment the initially "hot" ions with an injected beam diameter twice the diameter of the electron beam have been successfully cooled in $\sim 6 \mathrm{~s}$. The detector measurements have indicated an extremely low divergence angle of the cooled ions at about $3 \times 10^{-2} \mathrm{mrad}$ and a small momentum spread of only $(\Delta p / p)_{\mathrm{eq}}=5 \times 10^{-5}$.
In the TSR electron target the atomically clean $p^{+}$-GaAs photocathode, covered with a thin layer of cesium and oxygen, is cryogenically cooled down to $90 \mathrm{~K}$ and emits up to $1 \mathrm{~mA}$ of electrons with a reduced energy spread of about $7 \mathrm{meV}$, which is 15 times less than that of the thermocathode used in standard electron guns of cooler systems.

The ultracold electron beam has been obtained in the TSR experiment by adiabatic expansion of cold photoelectrons in the guiding magnetic field of the cooler. The transverse and longitudinal temperatures of photoelectrons

TABLE IV. Parameters during the TSR experiment and for the USR when operating with an internal target

\begin{tabular}{|c|c|c|}
\hline Ring & TSR & USR \\
\hline Ion & $\mathrm{CF}^{+}$ & $\bar{p}$ \\
\hline$A / Z$ & $31 / 1$ & $1 /-1$ \\
\hline Ion energy, keV/A & 97 & $300-20$ \\
\hline Ring circumference, $\mathrm{m}$ & 55.4 & 42.598 \\
\hline Horizontal/vertical betatron tunes & $2.84 / 2.77$ & $2.567 / 1.576$ \\
\hline$\beta=v / c$ & $1.4 \times 10^{-2}$ & $2.5 \times 10^{-2}-6.5 \times 10^{-3}$ \\
\hline Rotation period, $\mu \mathrm{s}$ & 13 & $5.6-21.8$ \\
\hline Vacuum pressure (hydrogen), Torr & $10^{-11}$ & $10^{-11}$ \\
\hline Injected beam intensity, ions & $\sim 2 \times 10^{4}$ & $2 \times 10^{7}$ \\
\hline Ion lifetime, s & 4 & $>10^{6}$ \\
\hline Initial/equilibrium emittance ( $\mathrm{rms}$ ), $\pi \mathrm{mm} \mathrm{mrad}$ & $1 / 10^{-3}$ & $5 / 0.3$ \\
\hline Equilibrium emittance low-beta mode (rms), $\pi \mathrm{mm} \mathrm{mrad}$ & & $0.7-1$ \\
\hline Initial/equilibrium & $2 \times 10^{-4} / 5 \times 10^{-5}$ & $10^{-3} / 6 \times 10^{-4}$ \\
\hline Equilibrium momentum spread (rms) low-beta mode & & $10^{-3}$ \\
\hline Ring acceptance, $\pi$ mm mrad & 120 & 100 \\
\hline Ring acceptance for low-beta mode, $\pi \mathrm{mm} \mathrm{mrad}$ & 40 & 30 \\
\hline Number of straight sections & 4 & 4 \\
\hline Length of achromatic straight section, $\mathrm{m}$ & 5.2 & 4 \\
\hline Periodicity: cooling mode & $2 \times 2(\mathrm{FP},-\mathrm{FP})$ & $4\left(D=D^{\prime}=0\right)$ \\
\hline Low-beta mode & $\begin{array}{l}\text { Antisymmetric } \\
\text { Electron cooler }\end{array}$ & $2\left(D=D^{\prime}=0\right)$ \\
\hline Length of electron cooler, $\mathrm{m}$ & $1.2(e$ target $)$ & 2 \\
\hline Electron beam energy, eV & 53 & $162-11$ \\
\hline Magnetic field at cooler, $\mathrm{kG}$ & 0.4 & $0.4-0.1$ \\
\hline Beta function at cooler (horizontal/vertical), m & $4 / 1.5$ & $7 / 7$ \\
\hline Dispersion at cooler, $\mathrm{m}$ & 2 & $D=D^{\prime}=0$ \\
\hline Friction force model & Parkhomchuk & Parkhomchuk \\
\hline Electron beam radius, $\mathrm{mm}$ & 6.5 & 10 \\
\hline Electron beam current, mA & $0.3\left(3 \times 10^{6}\right)$ & $1-0.05$ \\
\hline Adiabatic magnetic field expansion factor & 20 & 20 \\
\hline Cryogenic photocathode temperature $\left(\mathrm{kT}_{C}\right), \mathrm{meV}$ & 10 & 10 \\
\hline Electron temperature $\left(\mathrm{kT}_{\perp} / \mathrm{kT}_{\|}\right), \mathrm{meV}$ & $0.5 / 0.03$ & $0.5 / 0.045(162 \mathrm{eV}) 0.5 / 0.03(11 \mathrm{eV})$ \\
\hline Thermocathode temperature $\left(\mathrm{kT}_{C} \mathrm{meV}\right.$ & & 100 \\
\hline Thermocathode electron temperature $\left(\mathrm{kT}_{\perp} / \mathrm{kT}_{\|}\right), \mathrm{meV}$ & & $4 / 0.5$ \\
\hline \multicolumn{3}{|l|}{ 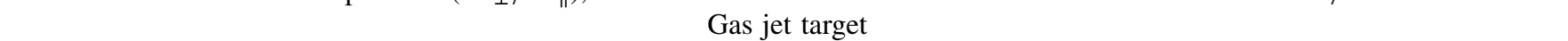 } \\
\hline Helium jet target density, $\mathrm{cm}^{-3}$ & & $5 \times 10^{11}$ \\
\hline Target diameter, $\mathrm{mm}$ & & 1 \\
\hline Beta function at target (horizontal/vertical), m & & $0.1-0.5$ \\
\hline Dispersion at target, $\mathrm{m}$ & & $D=D^{\prime}=0$ \\
\hline Cross section of $\mathrm{He}$ atoms ionization, $\mathrm{cm}^{-2}$ & & $5 \times 10^{7}$ \\
\hline Integral of ionization events & & $\approx 10^{7}$ \\
\hline
\end{tabular}


in the gun were estimated as $\mathrm{kT}_{\perp}=0.5 \mathrm{meV}$ and $\mathrm{kT}_{\|}=$ $0.03 \mathrm{meV}$, respectively.

The main limitations of the photocathode technique, such as degradation of the emission efficiency with time, have been overcome in the TSR electron target and the lifetime of the electron gun exceeds 24 hours. Photocathodes emit a rather small electron beam current compared to commonly used thermocathodes. But at the ultra-low-energy range the thermocathode performance is limited by the gun Perveance and the available electron current at $50 \mathrm{eV}$ beam energy is of the order of $I_{e}=$ 400-700 $\mu \mathrm{A}$; the emitted current from a thermoelectron gun at $10 \mathrm{eV}$ might thus not exceed $I_{e}=30-60 \mu \mathrm{A}$ [60]. Moreover, space charge effects in the electron beam limit the optimum electron beam current to the level available with photoguns. Thus, the photocathode performance itself and the emitted electron current are found to be satisfactory at low electron energies and close to the operating conditions of thermoelectron guns.

\section{B. Collapse of beam emittance at low beam intensities}

Simulations with BETACOOL have been benchmarked against measured data from the TSR with $\mathrm{keV}$ energy $\mathrm{CF}^{+}$ion beams and the results of these experiments have been successfully reproduced. We have studied the long-term beam dynamics and ion kinetics during beam circulation in the TSR storage ring. For the first time in the here-presented study, electron cooling has been added to the different heating processes that already included smallangle multiple scattering of the circulating ions on the residual gas atoms and molecules, IBS and ionization energy losses. Recombination was also taken into account by reducing the lifetime of $\mathrm{CF}^{+}$ions to $4 \mathrm{~s}$ as reported in [60]. Competing cooling and heating processes, the ratio between cooling and growth rates of beam emittance and momentum spread, beam intensity change as well as equilibrium conditions have all been investigated by simulating the rms parameters of the evolution of the ion distribution function over time. The theory of electron cooling is well developed and detailed information might be found at [62-64]. In our studies, the thin lens approximation of the electron cooler was chosen because both the variation in friction force, as well as the particle momentum change along the $1.2 \mathrm{~m}$ cooling section of the TSR, are small. The model of an electron gun of uniform cylinder shape with radius $\boldsymbol{r}_{e}$ and constant beam density $\boldsymbol{n}_{e}$ was used in our simulations.

In order to compare electron cooling experiments with computer simulations and benchmark BETACOOL at an ultra-low-energy range, we used the same parameters as reported above for the TSR experiment, see Table IV. Particles with a small emittance of about $\varepsilon_{x, y}^{\text {in }} \approx$ $1.5 \pi \mathrm{mmmrad}$ and low momentum spread of $(\Delta p / p)_{\text {in }}=2 \times 10^{-4}$ are injected into the TSR and accumulated using multiturn injection in combination with rf stacking $[65,66]$. In the TSR experiment with photoelectron cooling of low-energy $\mathrm{CF}^{+}$ions, a single turn injection of a low-intensity beam was employed. Thus, the initial parameters of the injected $\mathrm{CF}^{+}$beam, i.e., emittance $\varepsilon_{x, y}^{\text {in }}=1 \pi \mathrm{mm}$ mrad (rms), momentum spread $(\Delta p / p)_{\text {in }}=2 \times 10^{-4}(\mathrm{rms})$, and beam intensity of $N_{o}=2 \times 10^{4}$ ions, were chosen in our BETACOOL studies on beam shrinking.

The parameters of the standard TSR electron cooler are not optimized to reduce the already small initial emittance of the circulating beam of $\mathrm{CF}^{+}$ions. In our simulations we therefore used the parameters of the TSR cryogenically cooled photocathode with a transverse electron temperature of $\mathrm{kT}_{\perp}=500 \mu \mathrm{eV}$ and longitudinal electron temperature $\mathrm{kT}_{\|}=30 \mu \mathrm{eV}$, see Table IV.

We plotted the beam cross section of the circulating beam in the middle of the dispersion-free straight section of the ring just after injection into the ring but before cooling took place, see Fig. 13.

The rms widths of the beam are equal to $\sigma_{x}^{\mathrm{rms}}=2.2 \mathrm{~mm}$ and $\sigma_{y}^{\mathrm{rms}}=2.4 \mathrm{~mm}$ and the simulated beam spot before electron cooling is equal to $12 \times 20 \mathrm{~mm}^{2}$, which corresponds to experimentally measured beam dimensions [60].

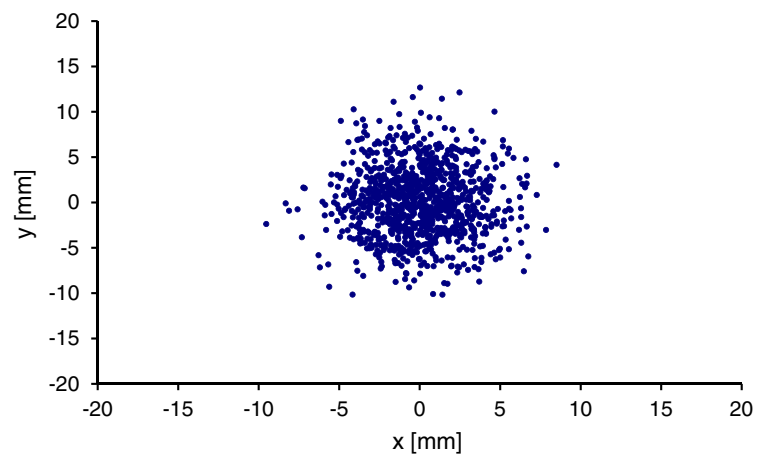

(a)

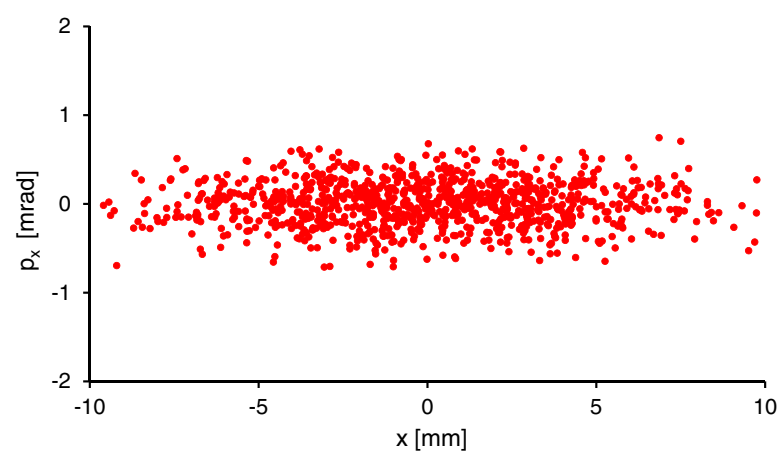

(b)

FIG. 13. Snapshots of a $\mathrm{CF}^{+}$beam injected into the TSR ring in the middle of straight section. Initial beam emittance is $\varepsilon_{x, y}^{\text {in }}=$ $1 \pi \mathrm{mm} \operatorname{mrad}(\mathrm{rms})$ : (a) simulated beam spot in the $x-y$ cross section where $\sigma_{x}^{\mathrm{rms}}=2.2 \mathrm{~mm}$ and $\sigma_{y}^{\mathrm{rms}}=2.4 \mathrm{~mm}$; (b) beam image in $x-x^{\prime}$ phase space corresponds to $4 \mathrm{rms}$ emittance $\varepsilon_{x, y}^{\text {in }} \approx 6 \pi \mathrm{mm} \mathrm{mrad}$. 


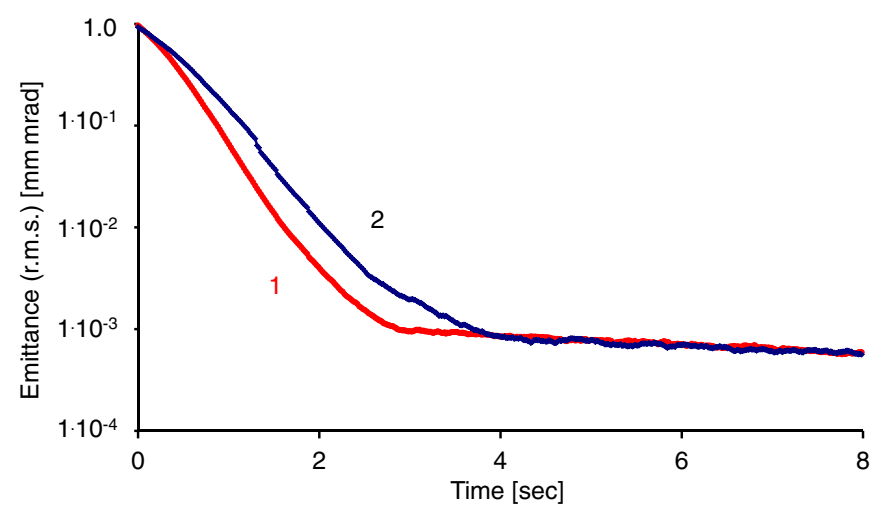

(a)

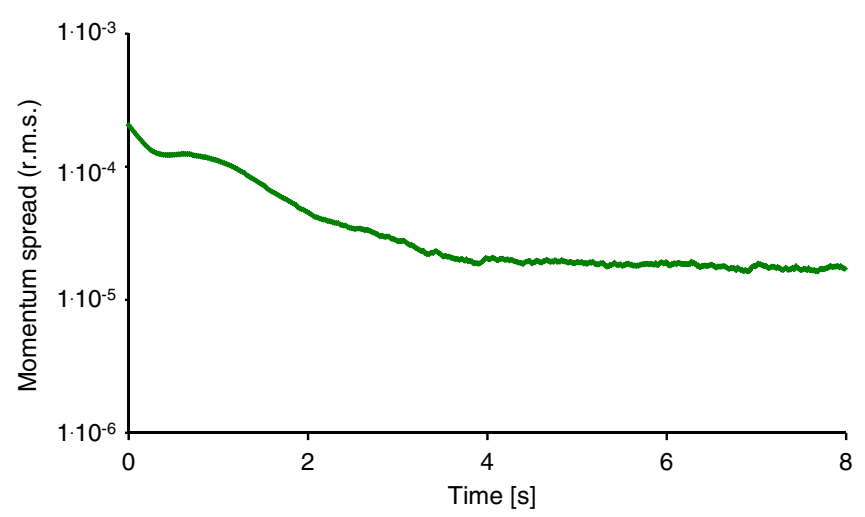

(b)

FIG. 14. Kinetics of $93 \mathrm{keV} / \mathrm{A} \mathrm{CF}^{+}$ions in the TSR ring: (a) shrinking of horizontal (1) and vertical (2) beam emittance by $\sim 1000$ times; (b) decrease of the momentum spread to equilibrium at $(\Delta p / p)_{\mathrm{eq}}=1.8 \times 10^{-5}$ (rms).

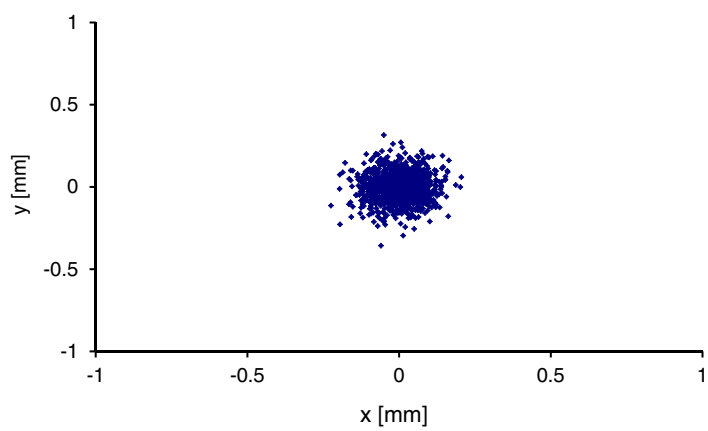

(a)

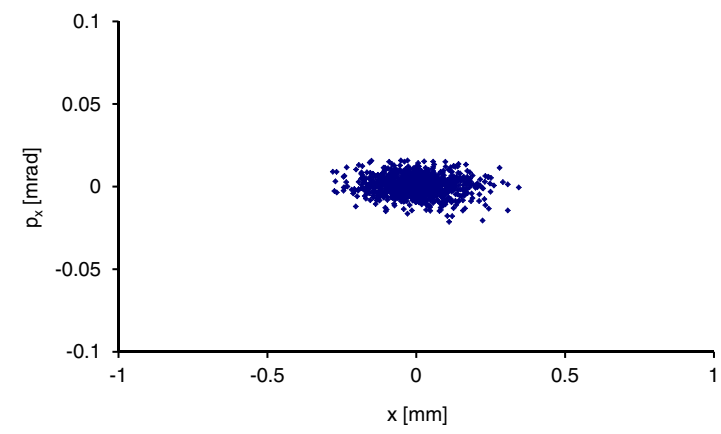

(b)

FIG. 15. Profiles of cooled $\mathrm{CF}^{+}$beam: (a) $x-y$ cross section in equilibrium, beam radius $\sim 0.2 \mathrm{~mm}$; (b) $x$ - $x^{\prime}$ phase space, the rms beam width $\sigma_{x, y}{ }^{\mathrm{rms}}=0.06-0.07 \mathrm{~mm}$ corresponds to equilibrium emittance $\varepsilon_{x, y}^{\mathrm{eq}}=6 \times 10^{-4} \pi \mathrm{mm} \mathrm{mrad}(\mathrm{rms})$.

A snapshot of the $\mathrm{CF}^{+}$injected beam in the $x$-x $x^{\prime}$ phase plane is also shown in Fig. 13(b). The area occupied by the beam in phase space corresponds to a 4 rms emittance of $\varepsilon_{x, y}^{\mathrm{in}} \approx 6 \pi \mathrm{mm} \mathrm{mrad}$. After two seconds of cooling time the beam emittance was reduced from $\varepsilon_{x, y}^{0}=1 \pi \mathrm{mmmrad}$ to an equilibrium at $\varepsilon_{x, y}^{\mathrm{eq}}=$ $10^{-3} \pi \mathrm{mm} \mathrm{mrad}$ (rms), see Fig. 14(a), showing that the emittance of the low-intensity beam has been reduced by a factor of 1000. Cooling by low-energy spread electrons emitted by photocathode reduces the transverse phase space of a low-intensity beam at least 10 times more efficiently than by conventional thermocathode. The momentum spread of ions cooled by photocathode is reduced by a factor of 10 and equilibrium is reached at $(\Delta p / p)_{\mathrm{eq}}=1.8 \times 10^{-5}(\mathrm{rms})$, see Fig. 14(b). The estimated cooling time is $\tau_{\text {cool }}=0.3 \mathrm{~s}$. The equilibrium dimensions of the cooled $\mathrm{CF}^{+}$beam are presented in Fig. 15. The envelope of the cooled beam is approximately equal to $0.2 \mathrm{~mm}$, see Fig. 15(a), and the angular divergence is less than $3 \times 10^{-2} \mathrm{mrad}$, see Fig. 15(b). One may derive the $\mathrm{rms}$ beam width of $\sigma_{x, y}^{\mathrm{rms}}=$ 0.06-0.07 $\mathrm{mm}$ in the middle of the TSR straight section.
This corresponds to an equilibrium emittance of $\varepsilon_{x, y}^{\mathrm{eq}}=$ $6 \times 10^{-4} \pi \mathrm{mm}$ mrad (rms).

So far, the agreement with experimental data is excellent despite some minor differences in numbers which we would refer to the simplifications of the model, to the uncertainties of the initial beam parameters, and to the margins in estimating the longitudinal and transverse temperature of the photocathode electron target cooler.

\section{COMPENSATION OF IBS AND INTERNAL TARGET EFFECTS BY ELECTRON COOLING IN THE USR}

The transition processes and equilibrium conditions of stored ions under the combined action of electron cooling and multiple scattering of the beam on residual gas, IBS, and scattering on an internal gas jet target have all been investigated by using BETACOOL and the results from numerical simulations have been successfully benchmarked against experimental data. In a final step, feasibility studies into the ion kinetics and long-term beam dynamics of ultra-low-energy ions have been performed on the example 
of the USR; a future facility designed to operate with antiprotons as well as with negatively and positively charged ions [67] in the $\mathrm{keV}$ range.

The USR design has been completely reconsidered since its initial layout from 2005 [68,69]. In order to match different requirements from a large variety of proposed experiments, several modes of operation are foreseen in the ring [70-72]. The modified geometry of the USR ring is based on a fourfold symmetry split achromatic lattice with four $4 \mathrm{~m}$ long achromatic straight sections which gives the necessary flexibility to satisfy multiple boundary conditions. The linear machine lattice was developed using the MAD-X and TRACE3D software and was reported elsewhere [69-71].

The parameters of the ring lattice, electron cooling system, target, and beam are listed in Table IV. The gas jet target and other components of the reaction microscope, the different rf systems for the short bunch operation mode [70,71], the electron cooler, the decelerating drift tube, and the elements for fast/slow extraction [72] are all located in three straight sections. One of the straight sections is kept free for the possible inclusion of a merged positron ring [73]. The beam characteristics might be varied over a wide range to operate the machine in various regimes in the tune diagram and specifically to achieve achromatic conditions in all four long straight sections as well as modify ring lattice to a twofold symmetry low-beta mode during operation with reaction microscope.

BETACOOL was used to find and optimize the parameters of the USR lattice and electron cooling system. The parameters of the antiproton beam are predefined by the previous chain of accelerators as well as by the efficiency of the antiproton production target [74]. The energy of the antiprotons injected into the USR is fixed at $300 \mathrm{keV}$. It is anticipated that the emittance of the injected beam will be about $\varepsilon_{x, y}^{\text {in }}=5 \pi \mathrm{mm} \mathrm{mrad}(\mathrm{rms})$ and the initial momentum spread about $(\Delta p / p)_{\text {in }}=10^{-3}(\mathrm{rms})$. The intensity of the ions circulating in the USR can be varied widely between $N_{0}=10^{4}$ and $2 \times 10^{7}$ ions [68].

We have checked the electron cooler performance for different initial conditions, such as at high and low-ion beam current, and at different ring settings. The ring, cooler, and target parameters are also listed in Table IV. An operation mode with a fourfold symmetry lattice is applied during injection, cooling, and deceleration. In this mode the high-intensity ( $N_{o}=2 \times 10^{7}$ ions) beam of $300 \mathrm{keV}$ ions can be cooled by a thermoelectron gun to equilibrium conditions corresponding to an emittance value of $\varepsilon_{x, y}^{\mathrm{eq}}=0.055 \pi \mathrm{mm}$ mrad (rms) and a momentum spread of $(\Delta p / p)_{\mathrm{eq}}=2.5 \times 10^{-4}(\mathrm{rms})$, see Fig. 16 .

We have also compared the cooling rates and equilibrium conditions of the USR beam cooled by an electron gun with a thermocathode with those cooled by a cryogenic photocathode $[60,75]$. The evolution of the rms emittance of a high-intensity beam of $N_{0}=2 \times 10^{7}$ antiprotons,

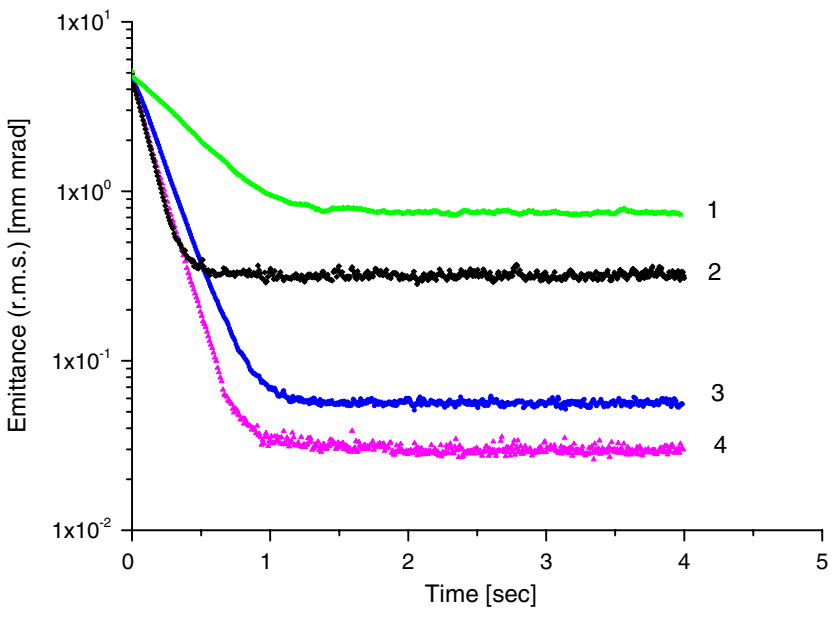

FIG. 16. Ion kinetics of a high-intensity antiproton beam $\left(N_{0}=2 \times 10^{7}\right)$ : (a) evolution of $20 \mathrm{keV}$ antiprotons cooled by (1) thermocathode $e$-gun to equilibrium emittance $\varepsilon_{x}^{\mathrm{eq}}=$ $0.73 \pi \mathrm{mm}$ mrad (rms) and cooled by (2) photocathode to equilibrium at $\varepsilon_{x}^{\mathrm{eq}}=0.35 \pi \mathrm{mm} \mathrm{mrad}(\mathrm{rms})$. Ions at $300 \mathrm{keV}$ cooled by (3) thermocathode to equilibrium emittance $\varepsilon_{x}^{\mathrm{eq}}=$ $0.055 \pi \mathrm{mm} \mathrm{mrad}(\mathrm{rms})$ and cooled by (4) photocathode to equilibrium at $\varepsilon_{x}^{\mathrm{eq}}=0.04 \pi \mathrm{mm} \mathrm{mrad}(\mathrm{rms})$.

cooled at 300 and $20 \mathrm{keV}$ is shown in Fig. 16 . Antiprotons cooled at $20 \mathrm{keV}$ are marked with (1) for the thermocathode gun and (2) for the photocathode gun. Antiprotons cooled by thermo- or by photocathode at an injection energy of $300 \mathrm{keV}$ are marked with (3) and (4), respectively. In the considered energy and current range, for low $\left(N_{0}=10^{4}\right.$ ions $)$ and high $\left(2 \times 10^{7}\right.$ ions $)$ beam intensities, the cooling rates and equilibrium parameters of a beam cooled by the photocathode are much better than those available with the thermocathode. At an injection energy of $300 \mathrm{keV}$, the high-intensity beam of antiprotons should be cooled by a photocathode to an equilibrium emittance of $\varepsilon_{x}^{\mathrm{eq}}=0.04 \pi \mathrm{mm} \mathrm{mrad}(\mathrm{rms})$, and by a thermocathode to equilibrium at $\varepsilon_{x}^{\mathrm{eq}}=0.055 \pi \mathrm{mm} \mathrm{mrad}$ (rms). At $20 \mathrm{keV}$ energy the equilibrium emittance of a beam cooled by photocathode is $\varepsilon_{x}^{\mathrm{eq}}=0.35 \pi \mathrm{mm} \mathrm{mrad}$ which is half the emittance of the beam cooled by thermocathode to equilibrium at $\varepsilon_{x}^{\mathrm{eq}}=0.73 \pi \mathrm{mm}$ mrad.

One might expect that the beam emittance should adiabatically grow from equilibrium value $\varepsilon_{x}^{\mathrm{eq}}=$ $0.04 \pi \mathrm{mm}$ mrad (rms) at $300 \mathrm{keV}$ (curve 4) to equilibrium value $\varepsilon_{x}^{\mathrm{eq}} \approx 0.16 \pi \mathrm{mm} \mathrm{mrad}(\mathrm{rms})$ at lowest beam energy, i.e., at $20 \mathrm{keV}$. BETACOOL simulations at $20 \mathrm{keV}$ provide a different value of equilibrium emittance $\varepsilon_{x}^{\mathrm{eq}}=$ $0.35 \pi \mathrm{mm}$ mrad (rms) (curve 2) which is twice the equilibrium emittance anticipated at $20 \mathrm{keV}$ due to adiabatic growth of the beam motion invariant alone. In these studies we did not take into account any beam mismatching, such as during rf capture or rebunching, or during deceleration. Thus, one may assume that the increased rate of intrabeam scattering at the lowest energy is one cause of the higher value of equilibrium emittance. 


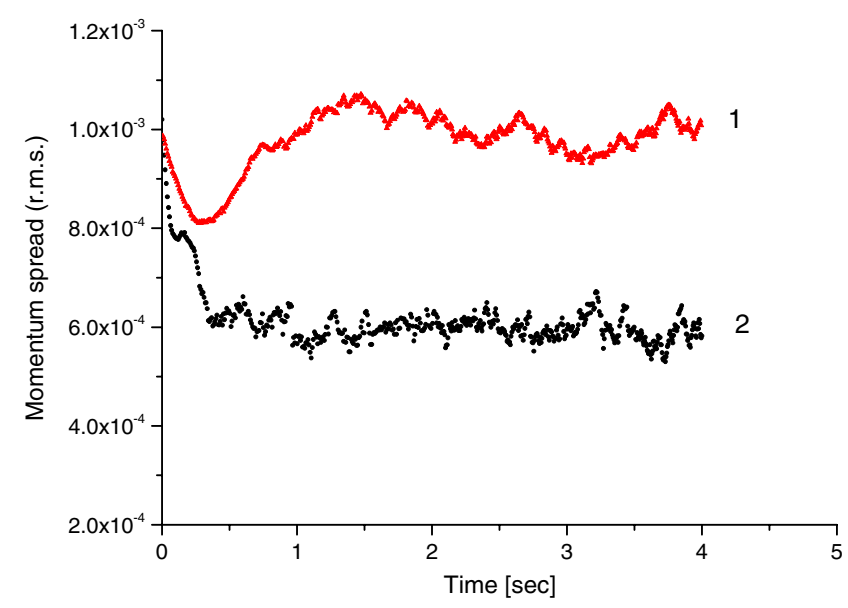

FIG. 17. Evolution of momentum spread (rms) of a highintensity beam of $20 \mathrm{keV}$ antiprotons cooled by an electron gun with thermocathode (1) and with photocathode (2). The equilibrium momentum spread is $(\delta p / p)_{\mathrm{rms}}^{\mathrm{eq}}=10^{-3}$ and $(\delta p / p)_{\mathrm{rms}}^{\mathrm{eq}}=6 \times 10^{-4}$, respectively.

The evolution of momentum spread (rms) of the highintensity beam with $N_{0}=2 \times 10^{7}$ ions of $20 \mathrm{keV}$ antiprotons cooled to equilibrium is presented in Fig. 17. Cooling by electron gun with thermocathode would lead to an equilibrium momentum spread of $(\Delta p / p)_{\mathrm{rms}}^{\mathrm{eq}}=10^{-3}$, while by photocathode equilibrium is reached at $(\Delta p / p)_{\mathrm{rms}}^{\mathrm{eq}}=6 \times 10^{-4}$.

Because of a better performance, we chose the parameters of the photocathode electron gun for further consideration. We also took into account the potential to conduct a technical realization of a simulated electron cooler with photocathode within the experiment.

Electron cooling of ultra-low-energy antiprotons will compensate for intrabeam scattering at high beam intensities of up to $N=2 \times 10^{8}$ ions as well as multiple scattering of ions on an internal target at high gas jet densities of up to $n_{\mathrm{tgt}}=10^{12} \mathrm{~cm}^{-3}$. We estimate that the number of useful ionization events might be as high as $1.5 \times 10^{7}$ counts during a $16 \mathrm{~s}$ operation cycle with an average count rate of about $\sim 20$ events per turn. We estimate that the beam lifetime will then be about $\tau_{\text {life }} \approx 12 \mathrm{~s}$.

\section{SUMMARY AND OUTLOOK}

In this paper we have presented the results from a comprehensive study into the beam behavior in low-energy storage rings. Nonlinear and long-term beam dynamics and ion kinetics were studied on the examples of ELISA, the antiproton decelerator antiproton recycler ring, the TSR in Heidelberg, and the USR. A consistent explanation of beam growth and associated ion losses based on benchmarking of ELISA experimental data was given. It was shown that the beam size in these rings is increased due to multiple scattering of ions on atoms and molecules of the residual gas, as well as due to intrabeam scattering at higher beam intensities. The main reason for ion losses at ultralow beam energies was identified in a reduced ring acceptance of nonlinear nature.

In addition, the requirements and conditions on ring operation with an internal gas jet target have been formulated. An achromatic lattice and low-beta mode of operation should be used in collision experiments with an internal gas jet target in order to provide a sufficient count rate of ionization events and reasonable beam lifetime. It was shown that operation with a dense gas jet target will be possible even at $\mathrm{keV}$ beam energies if these conditions are fulfilled. With a beam size on the target of around $1 \mathrm{~mm}$ and providing the initial intensity of the injected beam is more than $10^{7}$ antiprotons, it should then be possible to measure up to two counts of ionization events per turn in a recycler ring operating at the lowest energy of $3 \mathrm{keV}$ and up to seven events at a beam energy of $30 \mathrm{keV}$.

In addition, beam shrinking in the TSR experiment on electron cooling of low-energy $\mathrm{CF}^{+}$ions has been reproduced with good accuracy. The merits of photocathode guns for cooling of ultra-low-energy ions have been justified using both examples, TSR and USR. An optimization scheme of the USR ring lattice parameters has been formulated to improve the performance of electron cooling. It was shown that almost 20 ionization events per turn might be available in the USR ring during multiple crossings of $20 \mathrm{keV}$ antiprotons with a dense gas jet target.

Future beam dynamics studies will investigate further the effects of space charge on the stored beam, and in particular the evolution of the beam under consideration of the effects from electron cooling and an in-ring gas jet target.

\section{ACKNOWLEDGMENTS}

The here-presented work was supported by the Helmholtz Association of National Research Centers (HGF) and GSI Helmholtz Centre for Heavy Ion Research $\mathrm{GmbH}$ under Contract No. VH-NG-328, as well as the STFC Cockcroft Institute Core Grant No. ST/ G008248/1.

[1] L. H. Andersen et al., J. Phys. B 37, R57 (2004).

[2] P. Reinhed, Ph.D. thesis, Stockholm University, 2008.

[3] C. P. Welsch et al., AIP Conf. Proc. 796, 266 (2005).

[4] S. P. Møller, Nucl. Instrum. Methods Phys. Res., Sect. A 394, 281 (1997).

[5] T. Tanabe, K. Chida, K. Noda, and I. Watanabe, Nucl. Instrum. Methods Phys. Res., Sect. A 482, 595c (2002).

[6] S. Jinno, T. Takao, Y. Omata, A. Satou, H. Tanuma, T. Azuma, H. Shiromaru, K. Okuno, N. Kobayashi, and I. Watanabe, Nucl. Instrum. Methods Phys. Res., Sect. A 532, 477c (2004).

[7] K.-G. Rensfelt et al., in Proceedings of the 9th European Particle Accelerator Conference, Lucerne, 2004 (EPS-AG, Lucerne, 2004), pp. 1425-1427. 
[8] C.P. Welsch, J. Ullrich, C. Gläßner, K.-U. Kühnel, A. Schempp, H. Schmidt-Böcking, and R. Dörner, Phys. Rev. ST Accel. Beams 7, 080101 (2004).

[9] K. E. Stiebing et al., Nucl. Instrum. Methods Phys. Res., Sect. A 614, 10 (2010).

[10] D. Zajfman et al., J. Phys. Conf. Ser. 4, 296c (2005).

[11] M. A. El Ghazaly et al., Appl. Math. Inform. Sci. 3, 301 (2009) [http://naturalspublishing.com/files/published/ 86bdz3k479t84f.pdf].

[12] M. O. A. El Ghazaly et al., "Design of a novel electrostatic ion storage ring at KACST," Nucl. Instrum. Methods Phys. Res., Sect. A (to be published).

[13] S. P. Møller, in Proceedings of the 6th European Particle Accelerator Conference, Stockholm, 1998 (IOP, London, 1998), pp. 73-77.

[14] S.P. Møller, in Proceedings of the 18th Particle Accelerator Conference, New York, 1999 (IEEE, New York, 1999), pp. 2295-2297.

[15] S. P. Møller et al., in Proceedings of the European Particle Accelerator Conference, Vienna, 2000 (EPS, Geneva, 2000), pp. 788-790.

[16] Yu. Senichev and S. P. Møller, Proceedings of the European Particle Accelerator Conference, Vienna, 2000 (Ref. [15]), pp. 794-796.

[17] Yu. Senichev, FZJ IKP report, Jülich, 2000, p. 20.

[18] http://cosyinfinity.org.

[19] S. R. Mane, Nucl. Instrum. Methods Phys. Res., Sect. A 596, 288 (2008).

[20] F. Hinterberger, "Ion optics with electrostatic lenses," CERN School on Small Accelerators (2005), p. 24.

[21] OPERA-3D Reference Manual, Vector Fields/Cobham, England (2013).

[22] K. R. Crandall, Los Alamos National Laboratory Report No. LA-UR-90-4146.

[23] http://mad.web.cern.ch/mad.

[24] A.I. Papash and C.P. Welsch, in Proceedings of the International Particle Accelerator Conference, Kyoto, Japan (ICR, Kyoto, 2010), pp. 1952-1954.

[25] A. Gorda and A. Papash, QUASAR Group Design Note (2010), p. 5.

[26] A. O. Sidorin, I. N. Meshkov, I. A. Seleznev, A. V. Smirnov, E. M. Syresin, and G. V. Trubnikov, Nucl. Instrum. Methods Phys. Res., Sect. A 558, 325 (2006).

[27] BETACOOL Physics Guide [http://betacool.jinr.ru].

[28] I. Ben-Zvi et al., in Proceedings of the 21st Particle Accelerator Conference, Knoxville, 2005 (IEEE, Piscataway, NJ, 2005).

[29] I. Meshkov et al., Simulations of High-Energy Electron Cooling. Particle Accelerator Conference (Oak Ridge National Laboratory, Knoxville, 2005), pp. 4251-4253.

[30] A. V. Fedotov et al., in Proceedings of the 21st Particle Accelerator Conference, Knoxville, 2005 (Ref. [28]), pp. 4236-4238.

[31] I. Meshkov et al., Report No. RIKEN-AF-AC-21, 2000.

[32] I. Meshkov, D. Möhl, T. Katayama, A. Sidorin, A. Smirnov, E. Syresin, G. Trubnikov, and H. Tsutsui, Nucl. Instrum. Methods Phys. Res., Sect. A 532, 376 (2004).

[33] J. Dietrich et al., AIP Conf. Proc. 821, 154 (2005).
[34] C. Dimopoulou, K. Beckert, P. Beller, A. Dolinskii, U. Laier, F. Nolden, G. Schreiber, M. Steck, and J. Yang, Phys. Rev. ST Accel. Beams 10, 020101 (2007).

[35] A. Dolinskii et al., in Proceedings of the 9th European Particle Accelerator Conference, Lucerne, 2004 (Ref. [7]).

[36] B. Gålnander et al., AIP Conf. Proc. 821, 259 (2005).

[37] A. Noda, M. Ikegami, and T. Shirai, New J. Phys. 8, 288 (2006).

[38] A. Papash, A. Smirnov, and C. P. Welsch, in Proceedings of the International Particle Accelerator Conference, San Sebastian, Spain, 2010, pp. 2166-2168 [http:// www.jacow.org/].

[39] A. V. Smirnov, A. I. Papash, and C.P. Welsch, in Proceedings of COOL, Alushta, Ukraine, 2011 [http:// accelconf.web.cern.ch/accelconf/COOL2011/papers/ wecoa01.pdf].

[40] H. Risken, The Fokker-Planck Equation. Methods of Solution and Applications, Springer Series Edition (Springer, New York, 1996), p. 472

[41] V. Avilov, Solid State Commun. 44, 555 (1982).

[42] F. Hinterberger and D. Prasuhn, Nucl. Instrum. Methods Phys. Res., Sect. A 279, 413 (1989).

[43] E. Rutherford, Philos. Mag. 6, 21 (1911).

[44] G. Moliere, Z. Naturforsch. A2, 133 (1947).

[45] L. H. Thomas, Proc. Cambridge Philos. Soc. 23, 542 (1927).

[46] U. Fano, Phys. Rev. 93, 117 (1954).

[47] G. Moliere, Z. Naturforsch. 3A, 78 (1948).

[48] U. Fano, Annu. Rev. Nucl. Sci. 13, 1 (1963).

[49] H. A. Bethe, Phys. Rev. 89, 1256 (1953).

[50] A. Piwinski, in Proceedings of the 9th International Conference on High Energy Accelerators (1974), p. 105 [http://www.jacow.org/].

[51] M. Martini, Geneva Report No. CERN PS/84-9 AA, 1984.

[52] J.D. Bjorken and S. K. Mtingwa, Part. Accel. 13, 115 (1983).

[53] J. Wei, in Proceedings of the Particle Accelerator Conference, Washington, DC, 1993 (IEEE, New York, 1993), p. 3651.

[54] http://www.quasar-group.org.

[55] M. R. F. Siggel-King, A. I. Papash, H. Knudsen, M. H. Holzscheiter, and C. P. Welsch, Hyperfine Interact. 199, 311 (2011).

[56] J. Ullrich et al., Nucl. Instrum. Methods Phys. Res., Sect. B 124, 225 (1997).

[57] Knudsen, Helge et al. Phys. Rev. Lett. 101, 43201 (2008).

[58] A. Wolf et al., in Proceedings of COOL05 (2005), pp. 473-477 [http://www.jacow.org/].

[59] E. Jaeschke et al., in Proceedings of the European Particle Accelerator Conference, Rome, Italy, 1988, pp. 365-367 [http://www.jacow.org/].

[60] D. A. Orlov et al., Proceedings of COOL (Bad Kreuznach, Germany, 2007), pp. 230-233 [http://www.jacow.org/].

[61] D. A. Orlov, F. Sprenger, M. Lestinsky, U. Weigel, A. S. Terekhov, D. Schwalm, and A. Wolf, J. Phys. Conf. Ser. 4, 290 (2005).

[62] G. Budker, in Proceedings of the International Symposium on Electron and Positron Storage Rings, Saclay, 1966.

[63] I. N. Meshkov, Phys. Part. Nucl. 25, 631 (1994). 
[64] V. Parkhomchuk, Nucl. Instrum. Methods Phys. Res., Sect. A 441, 9 (2000), p. 9.

[65] G. Bisoffi et al., in Proceedings of the 1989 Particle Accelerator Conference, Chicago, IL (IEEE, New York, 1989), pp. 49-53.

[66] M. Steck et al., Proceedings of the European Particle Accelerator Conference (1988), pp. 1172-1174 [http:// www.jacow.org/].

[67] A. I. Papash, A. V. Smirnov, and C.P. Welsch, in Proceedings of the 3rd International Particle Accelerator Conference, New Orleans, Louisiana, USA, 2012 (IEEE, Piscataway, NJ, 2012).

[68] C. P. Welsch, M. Grieser, J. Ullrich, and A. Wolf Nucl. Instrum. Methods Phys. Res., Sect. A 546, 405 (2005).
[69] C.P. Welsch et al., Hyperfine Interact. 213, 205 (2012).

[70] A. I. Papash and C.P. Welsch, Phys. Part. Nucl. Lett. 6, 216 (2009).

[71] A. I. Papash and C.P. Welsch, Nucl. Instrum. Methods Phys. Res., Sect. A 620, 128 (2010).

[72] G. A. Karamisheva, A. I. Papash, and C. P. Welsch, Phys. Part. Nucl. Lett. 8, 50 (2011).

[73] I. Meshkov et al., in Proceedings of RuPAC, Russia, 2008 [http://www.jacow.org/].

[74] C. P. Welsch and J. Ullrich Hyperfine Interact. 172, 71 (2006).

[75] I. Meshkov (private communication). 